\title{
Evaluation of Dynamics of Fluttering and Autorotation of a Rigid Plate in a Flow Using Far-field Method
}

\author{
Ali Bakhshandeh Rostami*, Antonio Carlos Fernandes
}

COPPE, Federal University of Rio de Janeiro (UFRJ), Rio de Janeiro, Brazil

*Corresponding author, email: bakhshandeh@oceanica.ufrj.br

\begin{abstract}
The rotation of a non-circular body through a fluid flow is affected by different types of moment. In this paper, the total moment applied on the body into the fluid current is derived using far-field technique which uses the integral balance of the momentum equations. Such a complex expression of moment which is composed of many parameters is simplified in the case of a 2D rotating plate as moment due to pressure coefficient and moment due to vorticity field. Analytical expressions are obtained for each moment. The total torque on a rotating plate is decomposed to three parts included current effect, rotational effect, and mutual effect so that the last one is the contribution of the current velocity and the plate rotation simultaneously. The current effect represents the torque due to either the plate translates without rotation through a still fluid or the fluid passes over a stationary plate whilst the rotational effect deals with the torque generated by the pure rotation of the plate in the still fluid. To consider how different dynamical modes included fluttering (oscillatory rotation) and autorotation (continuous rotation) occurs on a 1DOF plate when is encountered by the current, we present a detailed discussion on the genesis and shed of vortices associated with each mode. We propose a condition related to torques by which the happening of each mode of rotation can be explained. Stability analysis shows that the transcritical bifurcation governs mainly on such physics however saddle node bifurcation might happen locally. A threshold is found for this physics.
\end{abstract}

Keywords: Fluttering, Autorotation, Vortex moment, Far-field method

\section{INTRODUCTION}

Interaction between boundaries and vortices (commonly uses "vorticity field") is an important problem not only in fluid-structure interaction because of inducing a motion into structures, i.e. buffet (Armandei et al, 2016), gallop (Rostami \& Armandei 2017), flutter (Samadpour et al 2016, Mehri et al, 2016, Asadi \& Wang 2017 a, 2017 b and Asadi et al, 2017) and so on, but also in vorticity and vortex dynamics (Rostami et al, 2016 and Ghadimi et al, 2012). The boundaries include either the surface of a rigid or flexible body or an interface of two different fluids. Hence, this problem covers wide range of science from biology, e.g. fish swimming, insect fly, to physics, e.g. lift generation, turbulent flow, stratified flow, and engineering, e.g. buffeting and galloping in bridges, Vortex induced Vibration in cylindrical bodies, etc. In all cases, the boundaries are fundamental source of creation of the vorticity. For an incompressible flow, the boundaries are the unique source of vortex generation and vorticity creation in a conservative external body-force field (Wu \& $\mathrm{Wu}$ 1996). The complexity of vorticity field in a bounded flow is further than an unbounded flow because of the genesis of vorticity from a boundary and the reaction of created vorticity 
to the boundary, and hence, as stated by (Wu \& Wu 1996), the unbounded flows are merely idealized approximations in the most practical cases.

The classical near-field method and the far-field method are the two main methods for obtaining quantities such as forces and moment in an external flow problem from the integrating of the fundamental fluid dynamic equations on boundaries. The classical nearfield method refers to the integration of the pressure and shear stresses on a solid surface, while the far-field method calculates forces and moment using the integral balance of the momentum equations (Marongiu and Tognaccini, 2010). In other words, in far field method, forces acting on a body are supposed to be related to Lamb vector (the cross product of the vorticity by the velocity) in case of unsteady Navier-Stokes flow. In the far field method, dynamic quantities, either forces or moment, are decomposed in a contribution associated with the bound vorticity on the body and a contribution related to the free vorticity developing in the body wake. Therefore, far filed method makes possible for an explicit interpretation of the physical processes which are involved in forces and moment generation. To achieve a result from far filed method, Gauss and Stokes theorems are fundamental theorems for implementing this technique. To consider the effect of local dynamics on the performance of integrand, the moment form of the integrand is used instead of the spatial derivative form. The pioneer in this sort of transformation was Thomson (1883) and Lamb (1945), Batchelor (1967), Lighthill (1986), Saffman (1992) and Wu, Ma \& Zhou (2006) pursued and developed the Thomson idea which created several named. In order to unification, Wu, Lu \& Zhuang (2007) reported that these transformations can be called by a unique name as derivative moment transformation (DMT). In DMT, developed by Wu (1981; see also $\mathrm{Wu} 2005$ ), a general vorticity moment theory was formulated for the total force and moment which a moving body through a still incompressible fluid will experience. This theory expresses in terms of the rate of change of the first and second integrated moments of the vorticity (Wu, Lu \& Zhuang, 2007).

Wang et al (2013) considered the force on a stationary and flapping flat plate at low Reynolds number using the near field method and investigated the contribution of two leading-order terms: the Lamb vector integral for the vortex force and the acceleration term of fluid for the unsteady inertial effect. Their formula which has been verified by the result of direct numerical simulation (DNS) revealed that the fields of the vertically projected Lamb vector and vertically projected acceleration around a flapping wing are directly responsible to the lift generation. The Lamb vector $(\omega \times \mathrm{u})$ was studied by Yang et al, (2007) using steady vortex force theory which uses far-field technique for calculation. They reported that the Lamb vector plays main role in force generation over a slender delta wing at a large angle of attack. Marongiu and Tognaccini, (2010) made a comparison between far-field and near-field techniques and stipulated that the far-field techniques provide several features that make them more attractive than the near-field techniques. They described that the far-field method allows interpreting the process involved in the genesis of the force explicitly. Besides, the decomposition of the contribution of the bound vorticity on the body and the free vorticity developing in the flow wake to the total force generation is definable. 
As mentioned in the first paragraph, the vortex generation on the surface of a body can result in an inducing of motion to the body. This motion calls Vortex Induced Motion (VIM) which has different types like as buffeting, galloping, fluttering, and tumbling (Rostami \& Armandei 2017). The two last motions, i.e. fluttering and tumbling (which is also called as autorotation), were considered in different literatures for falling objects. In this regards, it is worthy to note an earlier work done by Willmarth et al. (1964) in which they reported an experimental phase diagram for different types of motion of free falling disks. As following similar works, Field et al. (1997) found, by experiments, a chaotic region wherein lies between fluttering and tumbling of falling objects, Smith (1971) presented qualitatively a similar phase diagram for falling plates, Andersen et al (2005) used the numerical simulation of 2D Navier-Stokes equations to study the dynamics of a falling rigid card in air, and Belmonte, Eisenberg \& Moses (1998) studied the effect of moment of inertia on the transition from fluttering to tumbling in a quasi-two-dimensional experiment. In another work in 2015, Kuznetsov (2015) published a paper in which he analyzed the different proposed models for falling bodies, especially the models presented by Andersen et al (2005) and Belmonte et al (1998). Following the last work, Rostami and Fernandes (2018) developed two mathematical models for 1 DOF plate into the fluid current according to two models which have been presented by Andersen et al (2005) and Belmonte et al (1998). Rostami and Fernandes found that, with similar initial conditions, Anderson model gives better result in analysis of fluttering and tumbling (autorotation) in comparison to Belmont model.

This paper aims to derive and evaluate an analytical expression of the torque applied on a non-circular cylinder places through the fluid flow because of insufficient investigations on this topic in the literatures. The objective of this paper is to give a simple but efficient analytical expression for the torque applied on a rotating object which is articulated in the fluid current. Such an object can experience fluttering and autorotation. The finding of the current paper is useful in several fields of engineering such as aerodynamics and wind engineering, (Iversen, 1979, Lugt, 1983, and Trchalik et al, 2008), and Mechanical engineering, (Rostami and Fernandes, 2017) and renewable energy harvesting either in a small scale or in a large scale, (Rostami and Fernandes, 2014, 2015a, 2015 b, Fernandes and Rostami, 2015, Bakhshandeh rostami, 2015, and Rostami and Armandei, 2017).

In this research, we derived a far-field form only in terms of velocity and its derivatives on the surfaces of the control volume. This expression is used to analyse the stability of rotation of a flat plate. The specific aim of this paper is to elaborating the moment portions in order to understand their effects on the dynamics of rotating plate. Hence, a condition is proposed in which determines how a freely rotating object in the current start oscillating (fluttering) or continue its rotation (autorotation).

This paper is organized as follows. The integral form of torque expression on a body through the fluid flow is derived in $\S 2$. This torque expression is considered particularly for a 2D rotational plate in $\S 3$. In this section, the mathematical formulation of each term of the total torque on a rotating plate is determined. In $\S 4$, the process of vortex generation and shedding is considered for oscillatory rotation (henceforth is called fluttering) and for continuous rotation (hereafter is called autorotation) and then we probe comparatively into 
the different parts of the circulation and the torque in these phenomena. In this section, we discuss about the condition in which fluttering and autorotation will happen to a body. The stability analysis which is presented in $\S 5$ investigates the effect of mass moment of inertia on the dynamics of the plate. Concluding remarks are given in $\S 6$.

\section{General Form of Exciting Torques on a Body in a Fluid Flow}

Moment acting on a volume is found by the angular momentum theory. Hereinafter, the moment and the torque are used for the same physical term in this paper. This section attempts to present a general equation for the torque over a rotating body irrespective of its geometry and structural flexibility. To this end, the procedure is started by expanding the expression of the rate of angular momentum and followed by evaluating each term of the expanded equation separately to reach a more simple form and, at the end of section, bringing together all these evaluated terms to make a general and, to some extent, simple expression for the torque of a body through a fluid flow.

In mechanics, the torque is defined as the rate of change of angular momentum $(T=$ $d L / d t$ ). The angular momentum (L) of a fluid in a control volume is formulized as (Batchelor, 1967):

$\vec{L}=\frac{\rho}{3} \int \vec{r} \times(\vec{r} \times \vec{\omega}) d V_{B}$

where $\vec{\omega}=\nabla \times \vec{u}$ is vorticity vector, $\mathrm{r}$ is position vector and $\mathrm{V}_{\mathrm{B}}$ is control volume which is shown in the following figure.

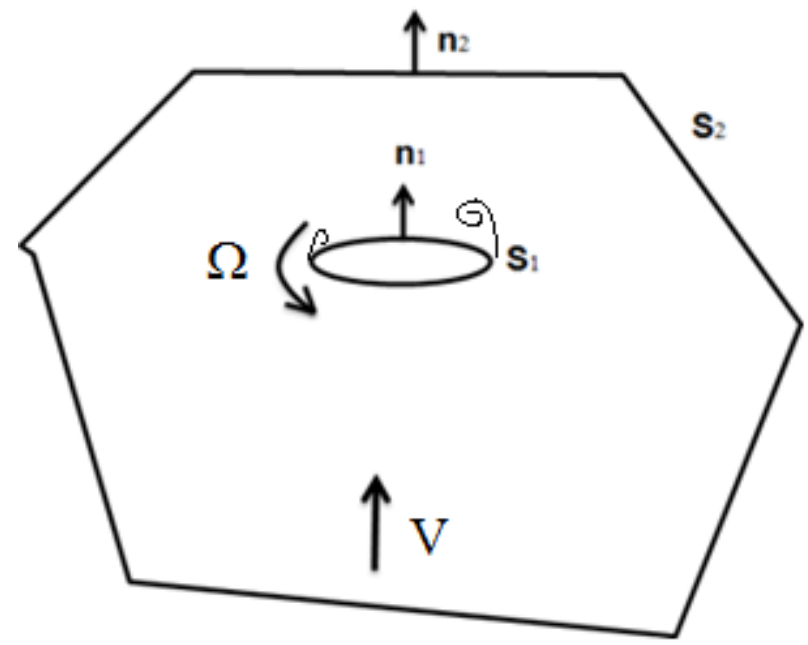

Figure 2-1: Control volume and control surface in the present study.

The torque is expressed as follow:

$\vec{T}=\frac{d \vec{L}}{d t}=\frac{\rho}{3} \int \frac{D}{D t}\left(\vec{r} \times(\vec{r} \times \vec{\omega}) d V_{B}\right.$ 
In equation $2.2, \frac{D}{D t}$ represents the material derivative which can be defined as $\frac{D \vec{A}}{D t}=\frac{\partial \vec{A}}{\partial t}+(\vec{u} . \nabla) \vec{A}$ where A is an arbitrary vector. Using this identity, equation 2.2 is written as:

$\vec{T}=\frac{\rho}{3}[\underbrace{\left(\int \frac{\partial}{\partial t}(\vec{r} \times(\vec{r} \times \vec{\omega})) d V_{B}\right)}_{\text {Unsteady }}+\underbrace{\int(\vec{r} \times(\vec{r} \times \vec{\omega}))(\vec{n} \cdot \vec{u}) d S}_{\text {Steady }}]$

where $\vec{n}$ is normal vector on the surface in outward direction. The equation 2.3 can also be obtained by Reynolds transport theorem (Wu, Ma \& Zhou, 2006). In the latest equation, the integrand of the steady part is evaluated as $(\vec{r} \times(\vec{r} \times \vec{\omega}))(\vec{n} . \vec{u})=\left[\vec{r}(\vec{r} \cdot \vec{\omega})-|r|^{2} \vec{\omega}\right](\vec{n} . \vec{u})$ using the well-known vector identity of $\vec{a} \times(\vec{b} \times \vec{c})=\vec{b}(\vec{a} \cdot \vec{c})-\vec{c}(\vec{a} \cdot \vec{b})$ where a, b and c are vectors. Accordingly, equation 2.3 yields:

$\vec{T}=\frac{\rho}{3}\left[\left(\int \frac{\partial}{\partial t}(\vec{r} \times(\vec{r} \times \vec{\omega})) d V_{B}\right)+\int \vec{r}(\vec{r} \cdot \vec{\omega})(\vec{n} \cdot \vec{u}) d S-\int\|r\|^{2} \vec{\omega}(\vec{n} \cdot \vec{u}) d S\right]$

Suppose that $u=d \vec{r} / d t$, distributing the time derivative to the unsteady part of equation 2.3 results in three easy-implementable integrals as below:

$\vec{T}_{\text {unsteady }}=\frac{\rho}{3}[\underbrace{\int \vec{u} \times(\vec{r} \times \vec{\omega}) d V_{B}}_{I}+\underbrace{\int \vec{r} \times\left(\vec{r} \times \frac{\partial \vec{\omega}}{\partial t}\right) d V_{B}}_{I I}+\underbrace{\int \vec{r} \times(\vec{u} \times \vec{\omega}) d V_{B}}_{I I I}]$

As corollary to the last equation, the unsteady torque over an object through the fluid flow is composed of the torque because of the unsteadiness in vorticity field, i.e. term II, and also the torque due to the interaction between the current and the vorticity field, i.e. terms I and III. The evaluation of each term is described in the following.

- Evaluation of term " $P$ "

The integrand of integral " $I$ " can be formed by $\overrightarrow{\mathrm{u}} \times(\overrightarrow{\mathrm{r}} \times \vec{\omega})=\overrightarrow{\mathrm{r}} \times(\overrightarrow{\mathrm{u}} \times \vec{\omega})-\vec{\omega} \times(\overrightarrow{\mathrm{u}} \times \overrightarrow{\mathrm{r}})$. On the other side, using the well-known vector identity of $\vec{a} \times(\vec{b} \times \vec{c})=\vec{b}(\vec{a} \cdot \vec{c})-\vec{c}(\vec{a} \cdot \vec{b})$, the term $\vec{\omega} \times(\overrightarrow{\mathrm{u}} \times \overrightarrow{\mathrm{r}})$ is broken down into two terms as $\vec{\omega} \times(\overrightarrow{\mathrm{u}} \times \overrightarrow{\mathrm{r}})=(\vec{r} \cdot \vec{\omega}) \vec{u}-(\vec{u} \cdot \vec{\omega}) \vec{r}$. Eventually, the integral " $I$ " in equation 2.5 is decomposed into three integrals as:

$\int \vec{u} \times(\vec{r} \times \vec{\omega}) d V_{B}=\int \vec{r}(\vec{u} \cdot \vec{\omega}) d V_{B}-\int \vec{u}(\vec{r} \cdot \vec{\omega}) d V_{B}+\int \vec{r} \times(\vec{u} \times \vec{\omega}) d V_{B}$

where, $\vec{u} . \vec{\omega}$ is called helicity which represents the projection of the particle spin at the direction of the motion (Saffman, 1992). The helicity measures the strengths of vortex filaments and their winding numbers (Wu, Ma \& Zhou 2006, p. 93). This parameter has been considered comprehensively in literatures (e.g. Moffatt 2014). In 2D flow, the vorticity vector is everywhere orthogonal to the plan of the flow which yields the helicity is identically zero, 
that is, the helicity is trivially conserved in 2D flow. The conservation of helicity in 2D flow has been proved by Yoshida and Morrison (2016).

The term of $\vec{u} \times \vec{\omega}$ in the last integral in equation 2.6 denotes Lamb vector which represents the Coriolis acceleration of a velocity field under the effect of its own rotation (Rousseaux et al, 2007). The volume integral of the Lamb vector is called the "vortex force" in the literatures for both of the incompressible and compressible flow (e.g. Marongiu \& Tognaccini 2010, Mele \& Tognaccini 2014, Wu Lu \& Zhuang 2007, Saffman 1992 and so on). Accordingly, the last integral of equation 2.6 calculates the moment on the body due to the vortex force.

- Evaluation of term " $I T$ "

The vorticity transport equation which is obtained by the curl of the momentum equation $\left(\nabla \times\left(\frac{D \vec{u}}{D t}\right)\right)$, is formulated as:

$\frac{\partial \vec{\omega}}{\partial t}=\nabla \times(\vec{u} \times \vec{\omega})+v \nabla^{2} \omega$

If an ideal barotropic fluid streams with the influence of conservative body forces, the vortex lines are transported with the fluid flow so that the vorticity flux is conserved across any Lagrangian surface element (Moffatt 2014). It means that, for an ideal barotropic fluid, the equation 2.7 reduces to Helmholtz equation $(\partial \vec{\omega} / \partial t=\nabla \times(\vec{u} \times \vec{\omega}))$. As corollary of Helmohotz equation, the term $\nabla \times(\overrightarrow{\mathrm{u}} \times \vec{\omega})$ in equation 2.7 is the only inviscid mechanism for the nonlinear vorticity transport. The second term of this equation $\left(v \nabla^{2} \omega\right)$ is known as the effect of viscous diffusion on the vorticity transport. As a result of viscosity, the vorticity in a flow tends to diffuse in space. For flows at large Reynolds numbers, the peak values of $v\left|\nabla^{2} \omega\right|$, carried by well-developed wake vortices, are quite weak (Wu Lu \& Zhuang, 2007). When Re»1, this happens inside the boundary layers, mainly in the sublayers adjacent to the body surface and right on the surface (Yang et al, 2007). In other words, in a high Reynolds number flow, the viscous diffusion of vorticity will be dominated by the other mechanism of the vorticity transport, i.e. the term $\nabla \times(\overrightarrow{\mathrm{u}} \times \vec{\omega})$ in equation 2.7 .

Aforesaid discussion leads to rearrangement of the integral " $I I$ ” in equation 2.5 as:

$$
\int \vec{r} \times\left(\vec{r} \times \frac{\partial \vec{\omega}}{\partial t}\right) d V_{B}=\int \vec{r} \times(\vec{r} \times \nabla \times(\vec{u} \times \vec{\omega})) d V_{B}+v \int \vec{r} \times\left(\vec{r} \times \nabla^{2} \omega\right) d V_{B}
$$

In addition, the integrals in the right hand side of the equation 2.8 are decomposed by the vector identity of $\vec{a} \times(\vec{b} \times \vec{c})=\vec{b}(\vec{a} \cdot \vec{c})-\vec{c}(\vec{a} \cdot \vec{b})$. Eventually, the torque generated by the vorticity variation (equation 2.8 ) is evaluated as: 


$$
\begin{aligned}
\int \vec{r} \times\left(\vec{r} \times \frac{\partial \vec{\omega}}{\partial t}\right) & d V_{B} \\
& =\int\|r\|^{2}(\vec{n} \times(\vec{u} \times \vec{\omega})) d S_{B}+\int \vec{r}(\vec{r} . \nabla \times(\vec{u} \times \vec{\omega})) d V_{B} \\
& +v \int \vec{r} \times\left(\vec{r} \times \nabla^{2} \omega\right) d V_{B}
\end{aligned}
$$

- Evaluation of term "III"

The Lamb vector is composed of gradient of velocity and advective velocity as follow:

$$
\vec{u} \times \vec{\omega}=\frac{1}{2} \nabla q^{2}-(\vec{u} . \nabla) \vec{u}
$$

where $q=|u|$ is Euclidean value of the velocity. The last integral of equation 2.5 is evaluated using equation 2.10 and integral form of divergence theorem.

$\rho \int \vec{r} \times(\vec{u} \times \vec{\omega}) d V_{B}=\rho \int\left[\vec{r} \times \frac{q^{2}}{2} \cdot \vec{n}+\vec{r} \times \vec{u}(\vec{u} \cdot \vec{n})\right] d S$

In the steady form of Bernoulli equation, the term $p=\frac{1}{2} \rho q^{2}$ expresses the dynamic pressure of the current on the body if the hydrostatic pressure is negligible (Salehi, Ghadimi $\&$ Rostami 2014). Denote $u_{n}=\vec{u} . \vec{n}$, then the second term in the right hand side is rewritten as $\vec{r} \times\left(\rho \vec{u} \mathrm{u}_{n}\right)$ which the term of $\rho \vec{u} \mathrm{u}_{n}$ represents the stress due to surface deformation (Wu Lu \& Zhuang 2006, pp.589 and pp.53-54). Now, by inserting equations 2.11, 2.9 and 2.6 into equation 2.4 , the total torque applied on a noncircular body through a fluid flow is acquired as:

$$
\begin{aligned}
\vec{T}=\frac{\rho}{3} \iint \vec{r}(\vec{u} \cdot \vec{\omega}) d V_{B}-\int \vec{u}(\vec{r} \cdot \vec{\omega}) d V_{B}+v \int \vec{r} \times\left(\vec{r} \times \nabla^{2} \omega\right) d V_{B} \\
\quad+\int \vec{r}(\vec{r} \cdot \nabla \times(\vec{u} \times \vec{\omega})) d V_{B}+\frac{2}{\rho} \int \vec{r} \times p \cdot \vec{n} d S_{B}+2 \int \vec{r} \times \vec{u}(\vec{u} \cdot \vec{n}) d S_{B} \\
\quad+\int\|r\|^{2}(\vec{n} \times(\vec{u} \times \vec{\omega})) d S_{B}-\int\|r\|^{2} \vec{\omega}(\vec{u} \cdot \vec{n}) d S_{B} \\
\left.+\int \vec{r}(\vec{r} \cdot \vec{\omega})(\vec{n} \cdot \vec{u}) d S_{B}\right]
\end{aligned}
$$

In general, regarding to equation 2.12, the total torque over a body through the flow comes from the helicity, the shear stress, the pressure distribution, the stress due to deformation and also the vorticity variation.

\subsection{Torque on a rotating 2D non-circular rigid body}

Consider a 2D rigid body placed into the incompressible fluid flow. In such a 2D problem, the velocity vector $(\vec{u})$ and the position vector $(\vec{r})$ are coplanar while the vorticity vector $(\vec{\omega})$ is normal to this plan. Suppose that $\vec{r}$ and $\vec{f}$ are coplanar vectors, then $\vec{r}(\vec{r} . \nabla \times \vec{f})=0$ (Wu, Ma \& Zhou 2006, pp.701). Thus, $\overrightarrow{\mathrm{r}}(\overrightarrow{\mathrm{r}} . \nabla \times(\overrightarrow{\mathrm{u}} \times \vec{\omega}))=0$ for the problems in 2D space. Being 
orthogonal of the vorticity vector $(\vec{\omega})$ to the velocity vector $(\vec{u})$ and the position vector $(\vec{r})$ results in $\vec{r} . \vec{\omega}=0$ and $\overrightarrow{\mathrm{u}} . \vec{\omega}=0$. As stated in forgoing section, for flows with high Reynolds number, the viscous diffusion of the vorticity transport goes to zero in far enough from the wall, that is, the $v \nabla^{2} \omega \cong 0$. Because the body is rigid, the surface deformation is negligible and the stress due to the surface deformation is negligible either, that is, $\rho \vec{u} \mathrm{u}_{n} \cong 0$ (Wu \& $\mathrm{Wu}, 1996)$.

By inserting the discussions given in the forgoing paragraph into equation 2.12, the total torque of a rotating $2 \mathrm{D}$ body with a rigid and non-circular section is found by:

$\vec{T}=\frac{2}{3} \underbrace{\int \vec{r} \times p \cdot \vec{n} d S_{B}}_{M_{\text {pressure }}}+\underbrace{\frac{\rho}{3} \int\|r\|^{2}(\vec{n} \times(\vec{u} \times \vec{\omega})) d S_{B}}_{M_{\text {vortex }}}$

In the last equation, $\mathrm{M}_{\text {pressure }}$ and $\mathrm{M}_{\mathrm{vortex}}$ represent the moment of pressure distribution and the moment generated by the vorticity field, respectively.

\section{Exciting torque of a rotating 2D rigid plate}

This section is devoted to reconsidering equation 2.13 to a rotating rigid plate in $2 \mathrm{D}$ space. Because the geometry of a flat plate is simple, therefore an analytical evaluation of each term in equation 2.13 is definable. Through this section, a procedure by which the expression of exciting torque on a flat plate is found is as follow: evaluation of pressure moment using the steady Blasius theorem, simplifying the formula of $\mathrm{M}_{\mathrm{vortex}}$ in equation 2.13 for a non-porous flat plate and finding a clear expression for this term, gathering together both aforementioned moment equations and forming the equation of dynamics of a 1 DOF rotating plate in order to finding the value of unknown coefficients in moment expressions, and eventually defining these unknown coefficients by fitting curves on experimental results.

\subsection{Evaluation of Moment of pressure}

One part of torque upon which a body places in the fluid flow is applied by the pressure distribution. Consider a rotating flat plate with an angular velocity of $\Omega$ through a flow with a speed of V. Suppose the plate is rotating around the center of rotation which places in the mid-chord, as shown in Figure 3-1. 


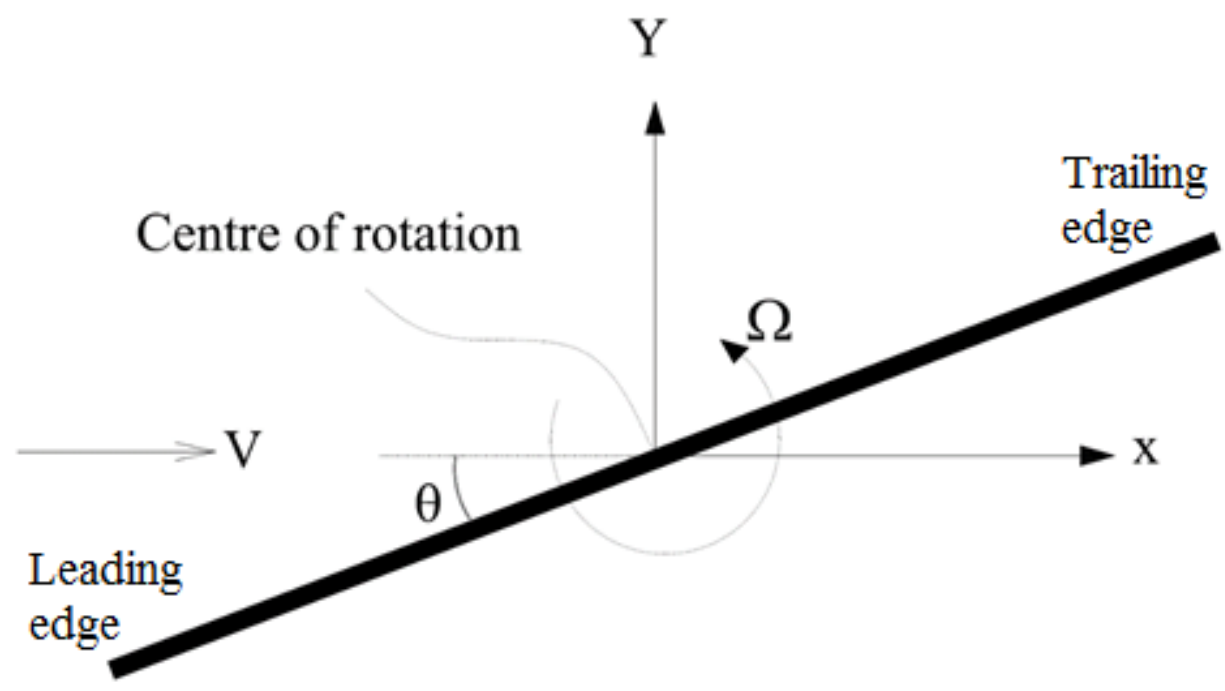

Figure 3-1: Schematic geometry of an articulated plate which is rotating in the fluid.

The rotational motion is the main difficulty of this problem. For a non-rotating body, it is easy to conformally map the body in the real plan ( $\mathrm{Z}$ plan) to a circle in the imaginary plan ( $\xi$ plan). Suppose a fixed non-circular cylinder into an irrotational and steady fluid current. The contour which closely encircled the stationary body is stream line, that is, the stream function around the cylinder in $\xi$ plan is constant. Accordingly, the moment due to the pressure distribution is calculated using the steady Blasius theorem given by equation 3.1:

$M_{\text {Pressure }}=$ Real part of $\left\{-0.5 \rho \oint z\left(\frac{d F(z)}{d z}\right)^{2} d z\right\}$

where $F(z)$ is a complex velocity potential of a fixed body in the z-plan which is found by Milne-Thompson circle theorem (Milne-Thompson, 1968). Milne-Thompson (1968) reported that the moment per length on an elliptic cylinder in the current of speed $\mathrm{V}$ with angle of attack $\theta$, is calculated by equation 3.2:

$M_{\text {Pressure }}=\frac{\pi}{2} \rho\left(a^{2}-b^{2}\right) V^{2} \sin 2 \theta$

where $\mathrm{a}$ and $\mathrm{b}$ are major and minor axis of the ellipse. Equation 3.2 indicates the moment on a stationary elliptical body while we know that the rotation affects the pressure distribution by changing the amount of velocity over the body surface. To tackle this difficulty, by keeping the general form of equation 3.2, the moment of pressure on a rotating elliptical body is expressed as:

$M_{\text {Pressure }}=\frac{\pi}{2} \rho f_{p}\left(a^{2}-b^{2}\right) V^{2} \sin 2 \theta$

where $f_{\mathrm{p}}$ is a dimensionless coefficient to determine rotational effect on the pressure distribution.

It is worthy to note that for a plate which is rotating with an angular velocity $\Omega$, the contour closely encircled the plate is no longer a stream line and it is impossible to use the 
circle theorem in global coordinate system (Xia \& Mohseni, 2013). However, in order to apply circle theorem in such a problem, Xia and Mohseni (2013) considered a non-inertial coordinate system which is stick on the plate and rotates with it. The moment of pressure of a rotating flat plate is calculated by equation 3.3 if set $a=C / 2$ and $b \approx 0$.

$M_{\text {Pressure }}=\frac{\pi}{8} \rho f_{p} C^{2} V^{2} \sin 2 \theta$

The coefficient $f_{\mathrm{p}}$ is determined as described it $\S 3.4$.

\subsection{Moment of Vortex Shedding}

Because of the velocity difference between the pressure and the suction side of the body through the fluid flow, vortices are formed where these velocities intersect. These vortices are called Leading Edge Vortex (LEV) and Trailing Edge Vortex (TEV). They can generate the moment on the body with non-circular cross section. This moment is expressed as:

$M_{\text {Vortex }}=\frac{\rho}{3} \int\|r\|^{2}(\vec{n} \times(\vec{u} \times \vec{\omega})) d S_{B}$

where $\overrightarrow{\mathrm{u}} \times \vec{\omega}$ is Lamb vector and $\|\mathrm{r}\|$ is Euclidean norm of the position vector $(\overrightarrow{\mathrm{r}})$. Using the vector identity, there is $\vec{n} \times(\vec{u} \times \vec{\omega})=\vec{u}(\vec{\omega} \cdot \vec{n})-\vec{\omega}(\vec{u} . \vec{n})$. If a solid wall is rotating or if a viscous tornado like vortex intersects an interface, viscous vortex tube cannot terminate at $S$ but will penetrate $\mathrm{S}$, that is, $\omega . n \neq 0$ (Wu \& Wu, 1996, pp,132). On the other hands, for non-porous walls, because of no-through condition, $\vec{u} \cdot \vec{n}=0$. When a non-porous rigid body rotates in a fluid flow then term of $\vec{n} \times(\vec{u} \times \vec{\omega})$ is simplified as $\vec{n} \times(\vec{u} \times \vec{\omega})=\vec{u}(\vec{\omega} \cdot \vec{n})$ and consequently equation 3.5 yields:

$M_{\text {Vortex }}=\frac{\rho}{3} \int\|r\|^{2} \vec{u}(\vec{\omega} \cdot \vec{n}) d S_{B}$

For a rotating rigid flat plate with angular velocity $\Omega$ in a fluid which flows with the velocity of $\mathrm{V}$, as shown in figure $3-1$, the point $\mathrm{P}$ somewhere on the body has the velocity $\vec{u}=\vec{V}+\vec{\Omega} \times \vec{r}$ where $r$ is the position vector referred to the axis of rotation and rigidly attached to the plate. By inserting the velocity vector into equation 3.6 and integrating over control surface, the vortex moment is determined as follow:

$M_{\text {Vortex }}=\frac{\rho}{3}\left(V C^{2} \Gamma+|\Omega| C^{3} \Gamma\right)$

where $\mathrm{C}$ is the chord length of the plate and $\Gamma$ is circulation which is defined, based on Stokes theorem, as $\Gamma=\int \vec{\omega} \cdot \vec{n} d S$. The circulation is a concept that relatives to the frame of reference and is measured by an observer on the body (Milne-Thompson, 1968). Here, $\mathrm{S}$ is the area enclosed by the projection of closed circuit on a plan perpendicular to $\vec{\omega}$.

The circulation $\Gamma$ is unknown and needs to be determined to complete the equation. For a correct description of the problem, it is essential to determine the circulation dependence on the dynamical attribute. In the previous works, the circulation was assumed an arbitrary 
constant value by Mahadevan (1996) or was modeled using the kutta-Joukowski theorem in classical low angle of attack problems by Tanabe \& Kaneko (1994) and Belmont et al (1998). The circulation of a rotating body through the current is composed of two parts, one corresponds to the angular velocity $\Omega$ and the other is proportional to current velocity which obtains by the Kutta-Joukowski condition. Both proportions play important roles in correct evaluation of dynamics of fluttering and autorotation on the body. Given to the KuttaJoukowsky lift at low angle of attacks, the circulation around a fixed non-circular thin object is defined as $\Gamma=\pi C V \sin \theta$ (Lamb, 1945). For taking into account the effect of the angle of attack rather than stall angle, this formula is written as $\Gamma=f_{T} C V \sin \theta$ where $f_{T}$ is a nondimensional coefficient which might be non-constant for a moving body. The rotational part of the circulation is formulated as $\Gamma_{R}= \pm f_{R} C^{2} \Omega$ (Sedov, 1980) in which $f_{R}$ is also a dimensionless coefficient. The sign of the rotational term depends on the direction of rotation, that is, it depends on the sign of the angular velocity. Thus, the total circulation around a non-circular rotating body is

$\Gamma=\underbrace{f_{T} C V \sin \theta}_{\begin{array}{c}\text { Current } \\ \text { effect }\end{array}}-\underbrace{f_{R} C^{2} \Omega}_{\begin{array}{c}\text { Rotational } \\ \text { effect }\end{array}}$,

Consequently, the moment of vortex is obtained as:

$M_{\text {Vortex }}=\frac{\rho}{3}\left[\left(f_{T} \sin \theta-f_{R} \operatorname{sgn}(\Omega)\right) C^{4} V|\Omega|+f_{T} C^{3} \sin \theta V^{2}-f_{R} C^{5}|\Omega| \Omega\right]$,

where $f_{T}$ and $f_{R}$ need to be determined.

\subsection{Total Torque on a rotating flat plate}

The torque of such physical system consists of the moment of pressure (Equation 3.4) and the moment of vortex (Equation 3.9) according to Equation 2.13. Consider a 2D flat plat of chord $\mathrm{C}$ which rotates with angular velocity $\Omega$ through the current of velocity $\mathrm{V}$. The torque which is applied to such a plate is:

$T=\frac{\rho C^{3}}{3}\left[\left(f_{T} \sin \theta+\frac{\pi}{4} f_{p} \sin 2 \theta\right) V^{2}+\left(f_{T} \sin \theta-f_{R} \operatorname{sgn}(\Omega)\right) C V|\Omega|-C^{2} f_{R}|\Omega| \Omega\right], \quad 3.10$

In the next section, we will discuss how the coefficients $f_{p}, f_{T}$ and $f_{R}$ will be determined. To aim for comparing the equation 3.10 with other models, it is worth to point out the model of Copeland (Copeland 1994) and also the model of Kuznetsov (Kuznetsov 2015). Both models are based on the damped pendulum model in which the exciting moment in the both models is given by trigonometric function (i.e. $\sin 2 \theta$ ) while the damping moment is different. Copeland reported a nonlinear damping torque with $5^{\text {th }}$ order polynomial equation whereas Kuznetsov presented this term as a linear function. Furthermore, the mutual effect of the fluid velocity and the angular velocity of the object has been ignored in both models.

\subsection{Determination of unknown coefficients}

To determine the unknown coefficients, the curve fitting method was chosen. In this method, the $f_{p}, f_{T}$ and $f_{R}$ change to acquire the best fit when the results of theoretical model is 
compared to those from experiments. In order to execute this method, we have conducted experiments in current flume of the Laboratory of Wave and Current (LOC) at Federal University of Rio de Janeiro (UFRJ), hereinafter called LOC/COPPE/UFRJ. The experimental setup is shown schematically in Figure 3-2. The fluid properties are shown in Table 1. Important characteristics of the plate are listed in Table 2.

Table 1: The fluid properties for experiment.

\begin{tabular}{lc}
\hline \multicolumn{2}{c}{ Fluid properties } \\
\hline Fluid type & Fresh water \\
Density & $1000 \mathrm{~kg} / \mathrm{m}^{3}$ \\
Velocity range & $0.07-0.45 \mathrm{~m} / \mathrm{s}$ \\
Temperature & $20 \mathrm{deg}$ \\
Current depth & $0.5 \mathrm{~m}$ \\
\hline
\end{tabular}

Table 2: Characteristics of the experimented model.

\begin{tabular}{lc}
\hline \multicolumn{2}{c}{ Plate properties } \\
\hline Width & $0.3 \mathrm{~m}$ \\
Material & Aluminum \\
Thickness & $5 \mathrm{~mm}$ \\
Height & $0.6 \mathrm{~m}$ \\
\hline
\end{tabular}

In the experiments, the dynamical characteristics of a freely rotating plate, e.g. time series of angle of rotation and angular velocity and also angular acceleration were measured by a pack of wireless sensors. This pack is composed of Arduino Uno as a microcontroller, UM7 which is a compact sensor included tri-axial accelerometer, rate gyro and magnetometer, and two Xbee modules as wireless communication modules, was used in the measurement of plate rotational attributes (See Figure 3-2). The data transmission rate in UM7 was set at 100 $\mathrm{Hz}$ using binary mode. The wireless communication was undertaken by two Xbee-pro 802.15.4 series 1 (S1) modules which were configured to communicate with 19200 as its Baud rate. The pack of sensor was installed on the model of an articulated plate as shown in Figure 3-2. 

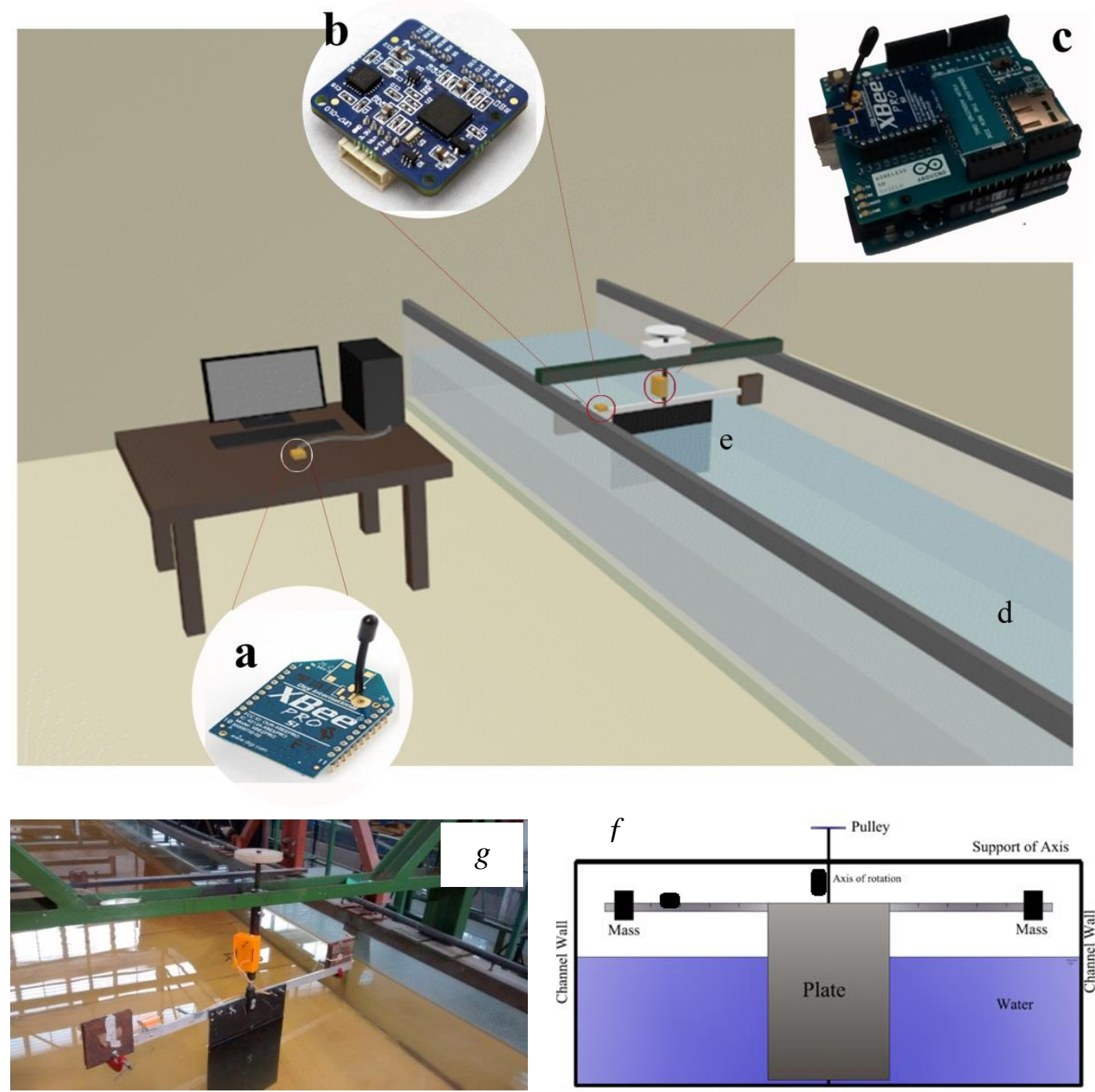

Figure 3-2: General view of experimental setup which is composed of (a): Xbee-pro 802.15.4 series 1, (b): UM7 sensor, (c): Assembly of the X-bee and the microcontroller (d): Current flume of LOC/COPPE/UFRJ (e): the setup of plate and instruments, (f): The setup of plate and instruments in front view (g): the model installed at LOC/COPPE/UFRJ.

Consider an articulated plate into the flow which rotates freely on the vertical axis places in the mid-chord. This axis is supported by bearings on the ends. The rotation of this system is defined by an ordinary differential equation (see equation 3.11) which is correspondent of the yawing motion.

$J \ddot{\theta}=\sum T(\ddot{\theta}, \dot{\theta}, \theta)=T^{\text {restoring }}(\ddot{\theta})+T^{\text {damping }}(\dot{\theta})+T^{\text {exciting }}(\dot{\theta}, \theta)$

Here, the dot symbol denotes the time derivative, that is, the $\ddot{\theta}=\dot{\Omega}$ and $\dot{\theta}=\Omega$ are the angular acceleration and the angular velocity, respectively. Moreover, $J$ is the structural mass moment of inertia of the plate. The right hand side of this equation consists of total torques applied on the plate including the exciting torque ( $T^{\text {exciting }}$ ) given by equation 4.1 , the restoring torque ( $T^{\text {restoring }}$ ) defined as $J_{A} \ddot{\theta}$ where $J_{A}$ is called added moment of inertia, and the damping torque ( $T^{\text {damping }}$ ) because of resistance in bearing components which is formulated as $D|\dot{\theta}|$. The added moment of inertia of a rotating elliptical cylinder is obtained 
by $J_{A}=\frac{1}{8} \pi \rho\left(a^{2}-b^{2}\right)^{2}$ where $\rho, a$ and $b$ are the fluid density, the major axis and minor axis of the ellipse, respectively. For a rotating flat plate with $\mathrm{a}=\mathrm{C} / 2$ and $\mathrm{b} \approx 0$, the added moment of inertia per chord length is calculated as $J_{A}=\pi \rho C^{4} / 32$.

Inserting the terms into equation 3.11 yields to theoretical equation of rotation of the flat plate with one degree of freedom:

$$
\begin{aligned}
\left(J+\frac{\pi \rho C^{4}}{32}\right) \dot{\Omega} & +\left(D-\left(f_{R} \operatorname{sgn}(\Omega)-f_{T} \sin \theta\right) \frac{\rho C^{4} V}{3}\right)|\Omega| \\
& =\frac{\rho C^{3}}{3}\left[\left(f_{T} \sin \theta+\frac{\pi}{4} f_{p} \sin 2 \theta\right) V^{2}-C^{2} f_{R}|\Omega| \Omega\right]
\end{aligned}
$$

where $\mathrm{D}$ is structural damping coefficient. The value of $\mathrm{D}$, for the current experimental setup, has been determined by the decay test as 0.033 [N.m.s]. Dimensionless form of the last equation is acquired using the length scale, $\mathrm{C}$, the velocity scale, $\mathrm{V}$, the time scale, $\tau=C / V$, the angular velocity scale, $\omega=\Omega C$, and the moment of inertia scale, $\epsilon=\rho C^{5}$. These characteristic scales construct five non-dimensional parameters as $\mathrm{t}^{*}, \lambda, \mathrm{I}^{*}$ and $\mathrm{D}^{*}, \mathrm{Re}$ which are named dimensionless time, tip speed ratio, dimensionless moment of inertia dimensionless structural damping, and Reynolds number, respectively and are formulated as:

$t^{*}=\frac{t V}{C}, \quad \lambda=\frac{\Omega C}{V}, \quad I^{*}=\frac{J}{\rho C^{5}}, \quad D^{*}=\frac{D \tau}{\epsilon}=\frac{D}{\rho V C^{4}}, \quad R e=\frac{V C}{\vartheta}$

In the previous equation, $\mathrm{D}^{*}$ is relevant to inverse of the current velocity. In other words, it is possible to state that $\mathrm{D}^{*}$ is proportioned inversely to Reynolds number as $\mathrm{D}^{*}=f\left(R e^{-1}\right)$. Using the characteristic scales and non-dimensional numbers, the following non-dimensional governing equation is obtained:

$$
\begin{aligned}
\left(I^{*}+\frac{\pi}{32}\right) \frac{d \lambda}{d t^{*}}+\left[D^{*}-\left(\frac{f_{R}}{3}\right.\right. & \left.\left.\operatorname{sgn}(\lambda)-\frac{f_{T}}{3} \sin \theta\right)\right]|\lambda| \\
& =\frac{f_{T}}{3} \sin \theta+\frac{\pi f_{p}}{12} \sin 2 \theta-|\lambda| \lambda
\end{aligned}
$$

To determine three unknown coefficients $\left(f_{T}, f_{R}\right.$ and $\left.f_{p}\right)$, the last equation is solved numerically by Adams-Bashforth method (see Lomax et al 2001) for given values of $I^{*}$ and $\mathrm{D}^{*}$. The unknown coefficients here play a tuning role and are defined to achieve the best curve-fitting with experimental data. Accordingly, the values of coefficients have been determined so that the uncertainty of determination is averagely about 1.5 percent. The values and their range of deviation are confronted as follow:

$$
\begin{aligned}
& f_{p} \approx \begin{cases}0.65 \pm 0.01 & \text { for fluttering } \\
0.82 \pm 0.01 & \text { for autorotation }\end{cases} \\
& f_{T} \approx \begin{cases}(0.101 \pm 0.001) \sin \theta & \text { for fluttering } \\
(0.115 \pm 0.001) \sin \theta & \text { for autorotation }\end{cases}
\end{aligned}
$$


$f_{R}=0.03 \pm 0.001 \quad$ for fluttering and autorotation

As can be seen, the coefficient $f_{T}$ is dependent on angle of attack. Similarly, Pesavento and Wang (2004) have reported a variable coefficient for circulation as $f_{T}=0.49 \cos \theta$. They have solved Navier-Stocks equation in $2 \mathrm{D}$ to capture total circulation around a falling elliptical cylinder. It is worthy to note that the solution of equation 3.14 using the cosine form of $f_{T}$ yields only the modeling of fluttering mode whereas, as shown in figure $3-3$, both modes of rotation (i.e. fluttering and autorotation) are properly modeled when the sine form is used.

The comparison of the theoretical model (equation 3.14) to the experimental data is shown in figure 3-3, for angle of rotation and angular velocity of fluttering and autorotation. The correspondent values of $\mathrm{I}^{*}$ and $\mathrm{D}^{*}$ in calculation are given in the caption of each figure. As can be seen from the figures, fluttering and autorotation can be modeled appropriately by the present theoretical model with the coefficients defined in equation 3.15. The results of fluttering mode demonstrate discrepancy with the experimental result. We ascribe such a discrepancy to the complexity of the vortex pattern in fluttering mode in comparison to this pattern in autorotation mode. In other words, the circulation model given in equation 3.8 might be slightly different when fluttering happens. However, this model is suitable for circulation of autorotation mode.

The period of rotation and the amplitude of angular velocity are other important criteria to ascertain whether the calculations are correct. It can be inferred from figures that the period of rotation and the amplitude of angular velocity are calculated with good accuracy. 

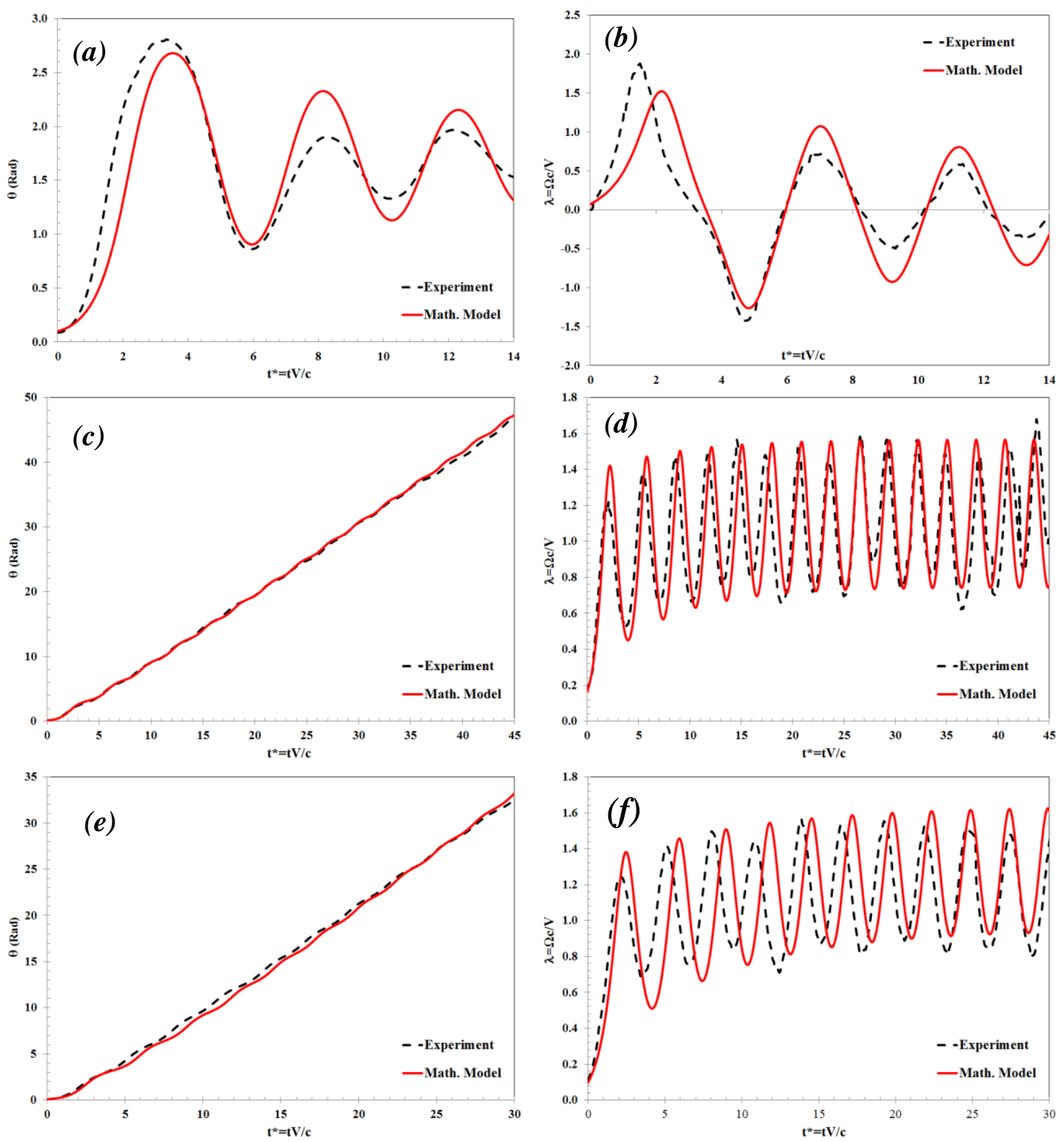

Figure 3-3: Comparison of non-dimensional results of angle of rotation $(\theta)$ and tip speed ratio $(\lambda)$ calculated by mathematical model (equation 3.14) and experimental data with respect to dimensionless time ( $t^{*}$ ). The values of parameters for calculation of diagram in $(a, b)$ are $I^{*}=0.0265$ and $D^{*}=0.0326$, in $(c, d)$ are $I^{*}=$ 0.132 and $D^{*}=0.01733$ and in $(e, f)$ are $I^{*}=0.151$ and $D^{*}=0.01322$.

\section{Discussion}

\subsection{Vortex generation and circulation}

According to Fernandes et al 2013, Rostami and Fernandes 2014, 2015a, 2015b and Fernandes and Rostami, 2015, two vortices which are called LEV and TEV are formed in each half-cycle of the rotation. Here, the TEV and the LEV represent the trailing edge vortex and the leading edge vortex, respectively. Figure 4-1 and Figure 4-2 illustrate sequence of generation, developing and shedding of LEV and TEV in a cycle of the fluttering and autorotation mode, respectively. As shown in these figures, the LEV starts forming at low angles of attack and keeps growing up to larger angles. The LEV is separated from the plate 
and shed to the current when it develops sufficiently. Meanwhile, the TEV forms at angle near which the LEV is shedding and starts developing.

Considering autorotation mode, by video processing over the experiments, the LEV is shed at an angle of approximately 130 degrees where the vortex had biggest radius which means that it was developed sufficiently. At this time, the TEV has not been developed properly and consequently is shed later at higher angle which is almost 170 degrees. Angles of shedding of the LEV and TEV are listed in table 4-1 for different I* at two Reynolds numbers. In fluttering mode, the shedding angles are determined in the first cycle of oscillation. The angle of shedding is appointed to angle at which a vortex separates from the plate. The uncertainty of the shedding angle is about 3 degrees, averagely. As can be concluded from table 4-1, the shedding angles are nearly independent of moment of inertia of the plate whereas in the contrary, they are dependent of the flow velocity. In other words, for different values of $\mathrm{I}^{*}$, the leading edge vortices (LEVs) shed at almost similar angles when the Reynolds number keeps constant. Increasing Reynolds number protracts the shedding of vortices, that is, for lower Reynolds numbers the vortices are separated from the plate at lower angles of attack.

Table 4-1: Angle of vortex shedding for LEV and TEV (the values are in degree),

\begin{tabular}{|c|c|c|c|c|c|}
\hline \multirow{2}{*}{$I^{*}$} & \multirow{2}{*}{$\begin{array}{c}\text { Mode of } \\
\text { rotation }\end{array}$} & \multicolumn{2}{|c|}{$\mathrm{Re}=5.39 \times 10^{4}$} & \multicolumn{2}{c|}{$\mathrm{Re}=9.98 \times 10^{4}$} \\
\cline { 3 - 6 } & & $\mathrm{LEV}$ & $\mathrm{TEV}$ & $\mathrm{LEV}$ & $\mathrm{TEV}$ \\
\hline 0.0186 & Fluttering & 126 & 58 & 132 & 63 \\
\hline 0.0321 & Fluttering & 124 & 58 & 131 & 61 \\
\hline 0.1216 & Autorotation & 125 & 165 & 132 & 171 \\
\hline 0.1962 & Autorotation & 124 & 162 & 131 & 169 \\
\hline
\end{tabular}

The shedding angles for the LEV and the TEV were reported as 160 and 170 degrees respectively by Martinez-Vaques et al. (2010) at $\mathrm{Re}=66.9 \times 10^{4}$ and as 140 and 170 degrees respectively by Smith (1971) at $\mathrm{Re}=9 \times 10^{4}$. Both reports were published using the results of the experimental study on an articulated flat plate into the wind tunnel whereas our experiments were conducted in the water channel. The discrepancy of shedding angles between our experiments and two aforementioned works can be ascribed to the difference in the shape of edges of the tested plate and also the difference in Reynolds number. Note that the angle of shedding of TEV for autorotation mode of a flat plate is nearly independent of Reynolds number as well as the moment of inertia, say that, the TEV is shed at $\theta \cong 170^{\circ}$ in different $I^{*}$ s and Res. 


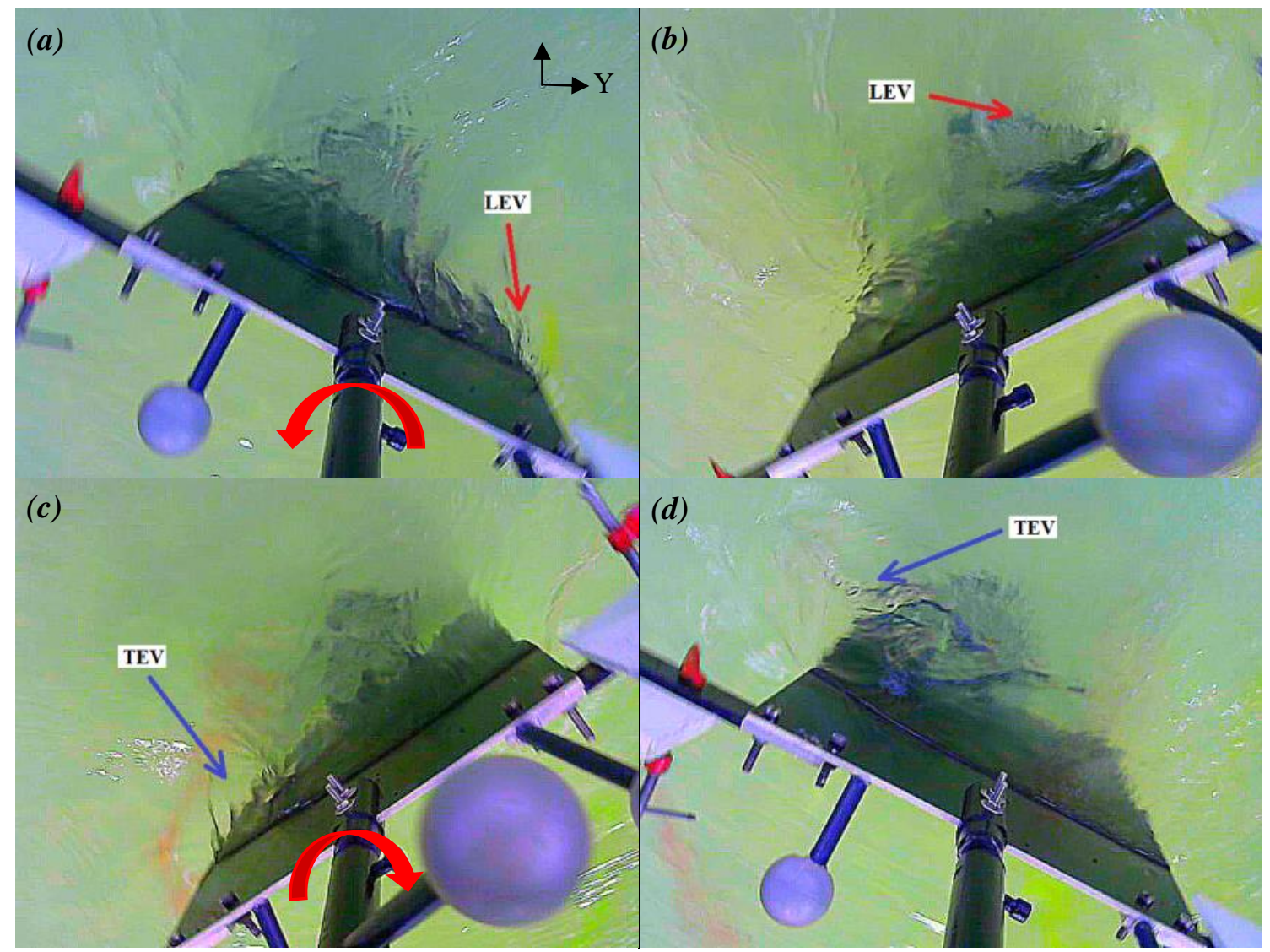

Figure 4-1: Dye visualization of vortex generation and shedding over the plate in fluttering mode (the view is along the vertical axis in the top to bottom direction): (a) the LEV formed and angular momentum of the plate begins increasing rapidly (the rotation direction is counter clockwise), (b) the LEV developed sufficiently and starts separating and the angular momentum is decreasing (the angular velocity is almost zero and this figure shows the maximum angle of oscillation), (c) the TEV is recognized clearly and it opposite angular momentum affects the direction of rotation which is reversing (the rotation direction is clockwise), $(\boldsymbol{d})$ angle of shedding at which the TEV reaches the maximum development and it is ready to separate (the angular velocity is almost zero and this figure shows the maximum angle of reversing oscillation). In these figures, curved arrows show the direction of rotation and red and blue line arrows refer the location of LEVs and TEVs. 


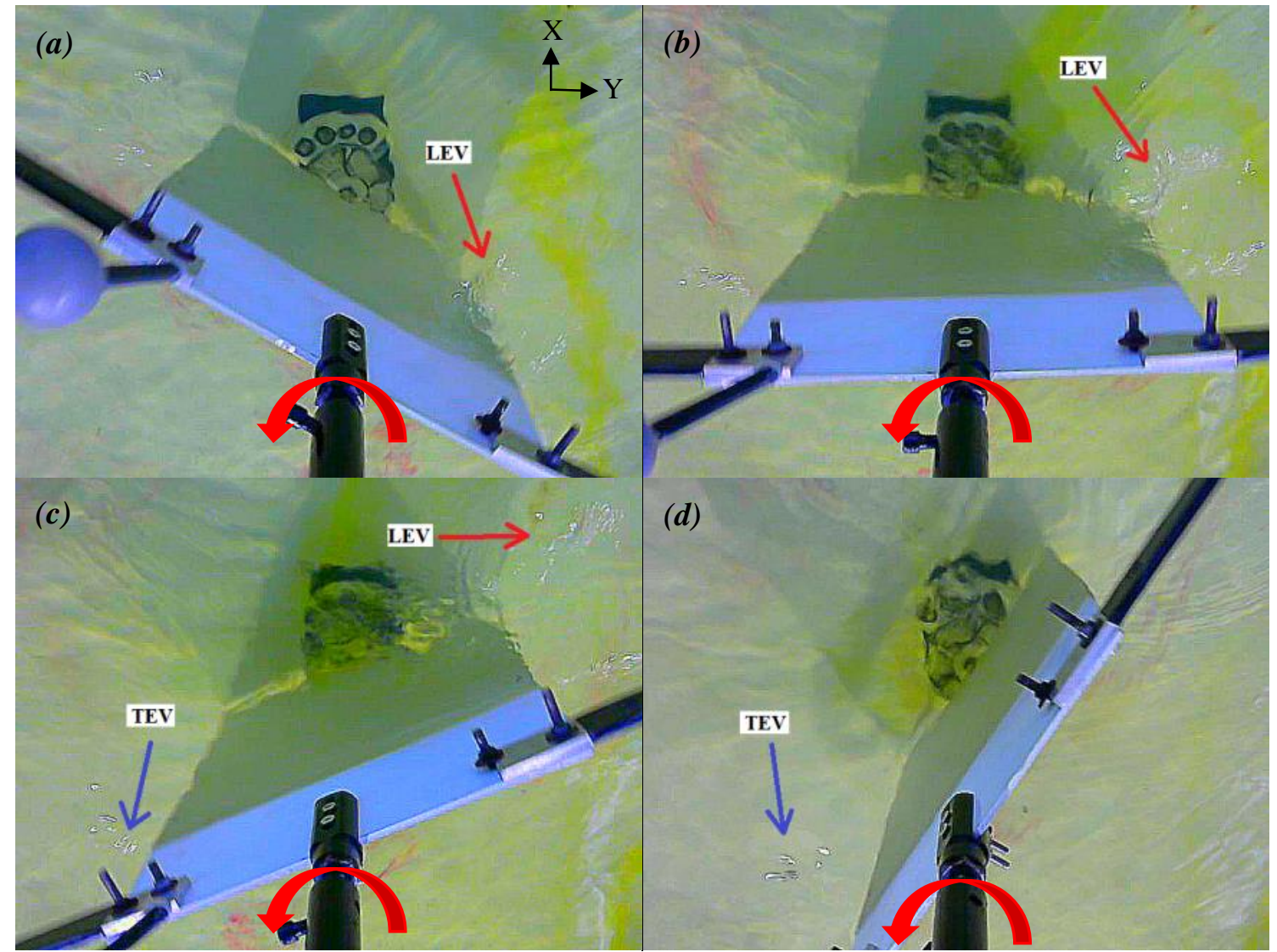

Figure 4-2: Dye visualization of vortex generation and shedding over the plate in autorotation mode (the direction of rotation is counter clockwise and the view is along the vertical axis in the top to bottom direction): (a) the LEV formed and is growing rapid, consequently, the plate angular momentum is growing quickly, $(\boldsymbol{b})$ the LEV is reaching to the maximum development with lower speed of growing, and as a result, the plate angular momentum decreases slowly $(\boldsymbol{c})$ the LEV is separating from the plate because of fully developed and the desired angular momentum is decreasing due to forming the TEV but the plate keep rotating in the same direction, $(\boldsymbol{d})$ the TEV sheds to the current and the plate keeps unidirectional rotation. In these figures, curved arrows show the direction of rotation and red and blue line arrows refer the location of LEVs and TEVs.

In these figures, the difference in size of TEV in fluttering and autorotation is considerable. The TEV develops properly in fluttering mode while in autorotation mode; it will shed without sufficient developing. In other words, the TEV in fluttering mode is bigger and stronger than the TEV in autorotation mode. Hence, fluttering happens because the TEV generates enough opposite angular momentum which pushes back the plate and resultantly, the oscillation starts. On the other side, because the LEV is greater in intensity than the TEV in autorotation mode (Iversen, 1979), a unidirectional rotation will occur. The circulations of the TEV and LEV have unequal values while their signs are also opposite, i.e. they rotate contrary to each other, LEV rotates counter clockwise while TEV rotates clockwise. The total circulation is computed by the sum of the circulation of both vortices at each cycle, and consequently the total cyclic circulation is non-zero.

Non-dimensional circulation $\left(\Gamma^{*}=\Gamma / V C\right)$ is depicted with respect to angle of rotation $(\theta)$ in figure 4-3. The circulation depends on the direction of rotation, accordingly, the rotational part of fluttering includes the negative and the positive values and it becomes small by the time because of the dissipating of the oscillation. The effect of rotation on the circulation is indicated by blue dashed line in figure 4-3. The contribution of the current effect in the total circulation of the fluttering mode increases while the rotational part vanishes. 


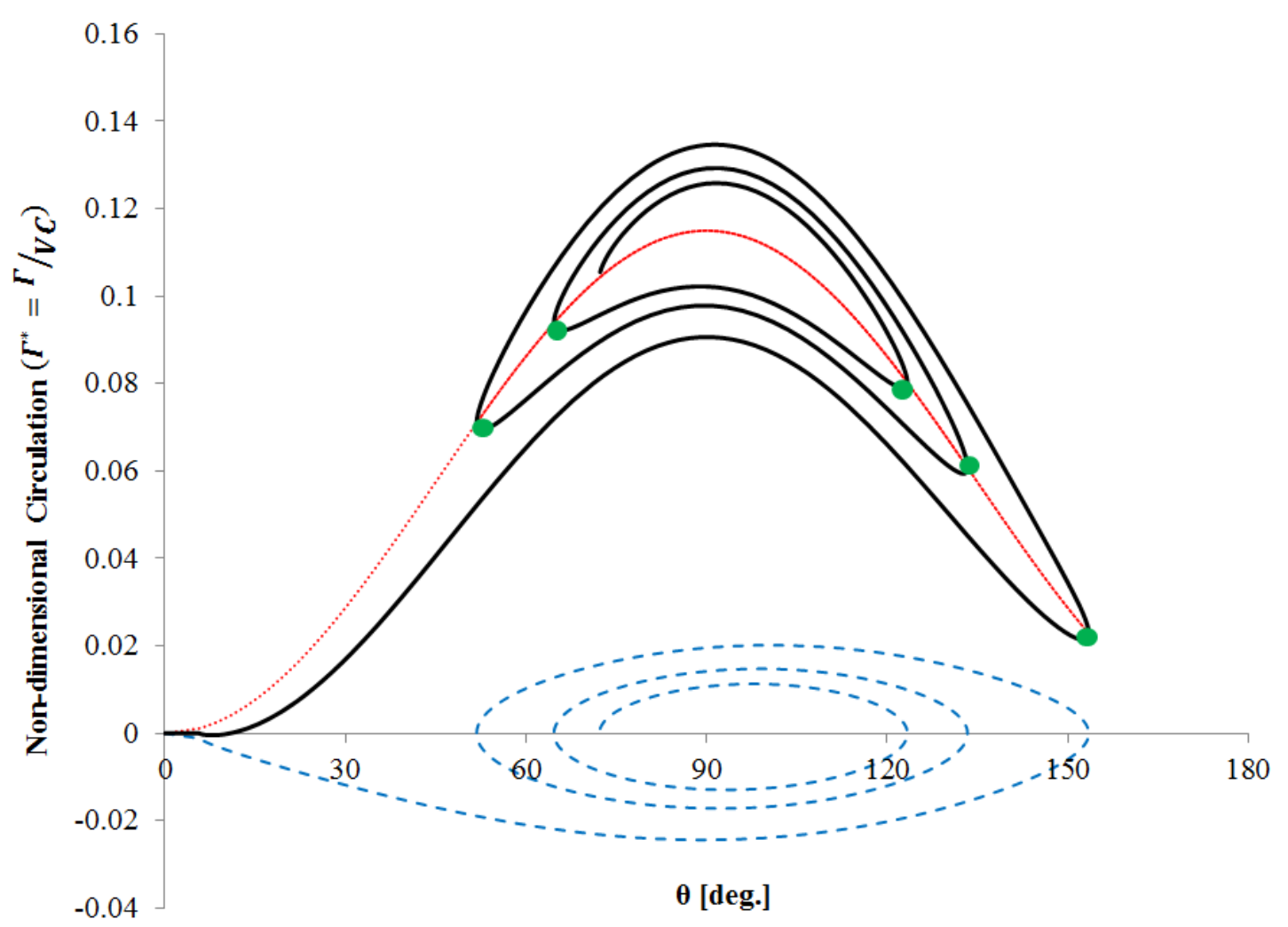

Figure 4-3: Non-dimensional circulation diagram with respect to angle of rotation for fluttering (oscillatory rotation). Red dotted line denotes the contribution of current effect while blue dashed line points out the contribution of rotational part in total circulation which is depicted by black solid line.

\subsection{Torque}

The LEV provides a convenient torque in the direction of rotation of the plate. Having known the shedding angles of LEV in autorotation and fluttering in the first cycle are equal, what is the source of occurring fluttering or autorotation in this plate? To answer this question, it is appropriate to elaborate the resisting torque which mainly provides by the TEV. Recall equation 3.10 which indicates the total torque over a 2D rotating flat plat. The total torque is composed of three components as indicated in equation 4.1.

$T=\frac{\rho C^{3}}{3}[\underbrace{\left(f_{T} \sin \theta+\frac{\pi}{4} f_{p} \sin 2 \theta\right) V^{2}}_{\text {current } \text { effect }}-\underbrace{C^{2} f_{R}|\Omega| \Omega}_{\begin{array}{c}\text { Rotational } \\ \text { effect }\end{array}}+\underbrace{\left(f_{T} \sin \theta-f_{R} \operatorname{sgn}(\Omega)\right) C V|\Omega|}_{\text {Magnus effect }}] \quad 4.1$

The value of coefficients $f_{p}, f_{T}$ and $f_{R}$ defined in equation 3.15. Three decomposed terms in equation 4.1 are: the first one is the current effect which represents the torque over the plate due to either the translation of the plate through a still fluid with velocity $\mathrm{V}$ or passing a fluid flow of velocity $\mathrm{V}$ over a stationary plate when the angular velocity is zero, the second term is about the rotational effect which calculates the torque generated by the rotation of the plate in the still fluid, and the third part is the contribution of the current velocity and the plate rotation simultaneously which is named Magnus effect. In the case of falling objects, as stated by Pessavento and Wang (2004), the Magnus term $(V \Omega)$ specifically is important to 
generate the sufficient lift at the turning points of the trajectory, where the translational velocity $\mathrm{V}$ is small while the angular velocity $\Omega$ increases, to elevate the center of mass.

Figure 4-4 shows the torque coefficient of Magnus and rotational effects indicated in equation 4.1. Although, the Magnus torque plays both roles as the exciting of rotation (when has a positive value) and the resisting of rotation (when the value is negative), but the rotational part is a resisting torque because of being ever negative. In a cycle, the value of Magnus torque becomes negative in a range of angles at which the LEV already shed to the current, i.e. the angles greater than the LEV shedding angles. After shedding the LEV and because of the existing angular momentum resulted by the LEV, the plate keeps rotating in the same direction till the angle at which the TEV angular momentum (resisting torque) overcomes the plate angular momentum. At this angle, the returning rotation is started and fluttering rotation emerges. In figure 4-3, the green points denote the aforementioned angles at which the angular momentum of the vortices (LEV and TEV) becomes greater than the plate angular momentum and consequently, the direction of rotation and also the angular velocity change.

In the case of autorotation, the rotation will continue in a given direction if an additional and sufficient large torque prevail the resisting torque when the LEV shed into the current. The additional torque is provided by the mass moment of inertia of the system which is called inertial torque, hereinafter. The inertial torque and its coefficient are defined as $T_{I}=U+$ $\left.\pi \rho C^{5} / 32\right) \dot{\Omega}$ and $C_{T_{I}}=2 C^{2}\left(I^{*}+\frac{\pi}{32}\right) \dot{\Omega} / V^{2}$, respectively. The rotation continues and makes autorotation only if the condition of $\left|C_{T_{I}}\right|>\left|C_{T_{R A}}\right|$ is met in the adjacent to the shedding angle. Here, $C_{T_{I}}$ is the coefficient of inertial torque and $C_{T_{R A}}$ is the coefficient of resisting applied torque which is calculated by equation 4.1 . The situation in which $\left|C_{T_{I}}\right|<\left|C_{T_{R A}}\right|$, the plate starts oscillating because it has not enough big angular momentum to overcome the resisting angular momentum. In figure 4-5, which presents the torque coefficient of fluttering motion in two first oscillations, the last condition governs at all indicated shedding angles. In other words, at all shedding angles, the absolute value of the inertial torque coefficient $\left(\left|C_{T_{I}}\right|\right)$ is less than the absolute value of the resisting applied torque coefficient $\left(\left|C_{T_{R A}}\right|\right)$.

It can be inferred from foregoing paragraphs that the moment of inertia plays a critical role for occurring fluttering and autorotation. It means that the stability of each mode depends on the value of moment of inertia of the system. 


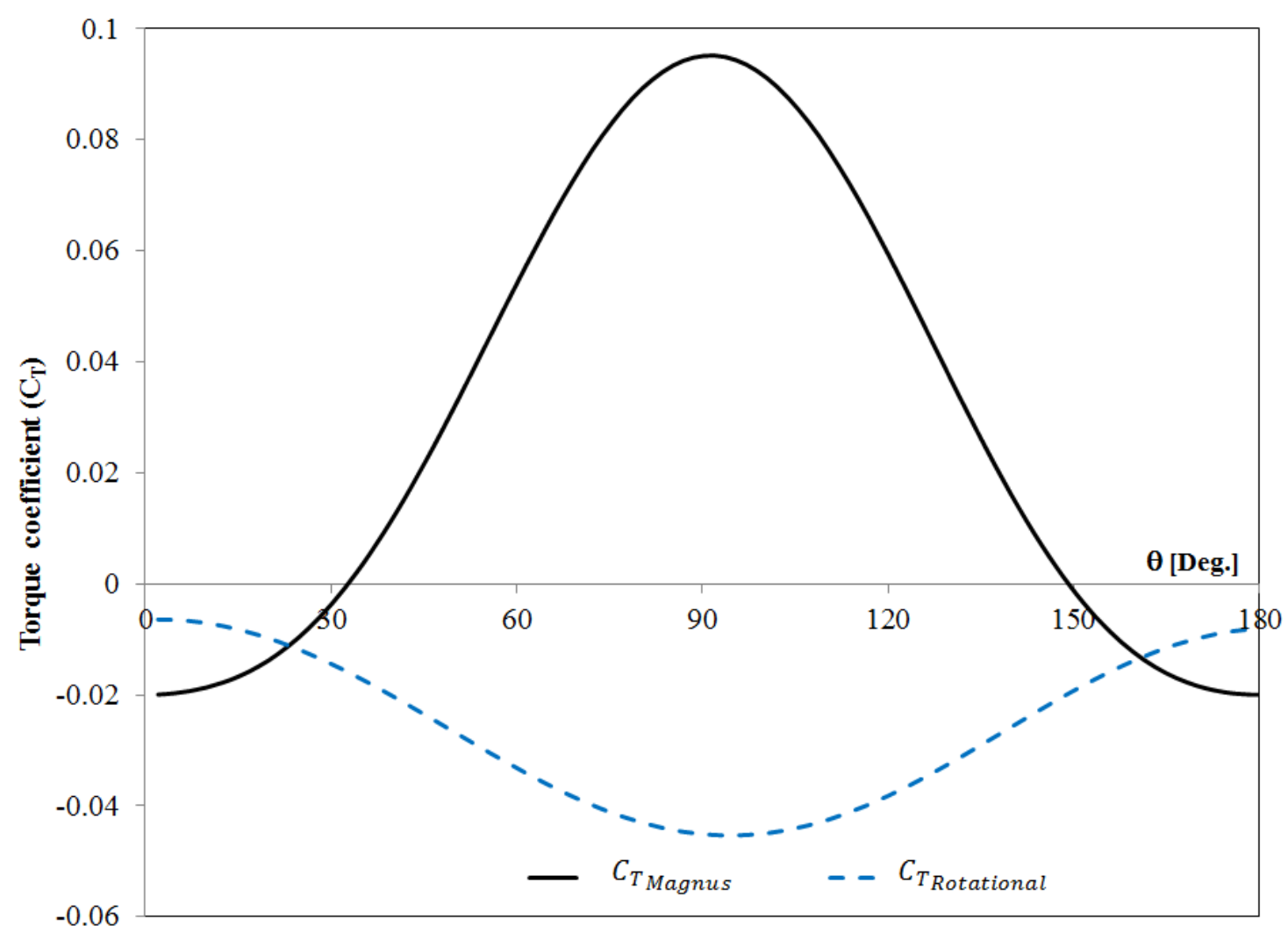

Figure 4-4: The coefficients of Magnus torque and rotational torque.

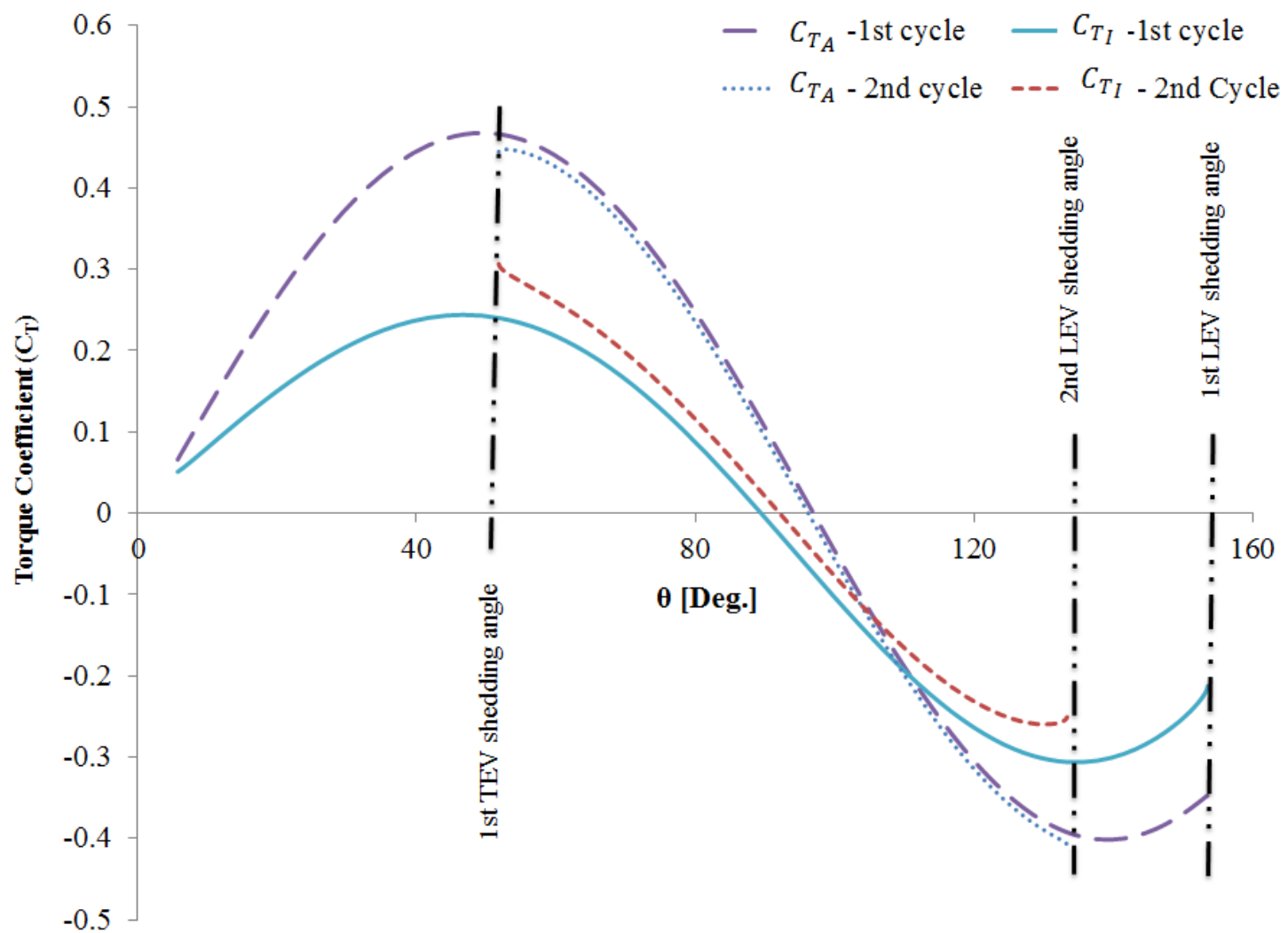

Figure 4-5: Inertial and applied torque coefficients on the plate for fluttering mode. The shedding angles related to LEV and TEV have been indicated on the figure and it is demonstrated that inertial torque is less than applied torque in fluttering mode at adjacent of the shedding angle. 


\section{Stability Analysis}

Recall equation 3.14 for stability analysis. It is necessary to find fixed points of the equation at first step. This equation has general form as:

$\dot{\lambda}=f\left(\frac{\lambda}{I^{*}+\frac{\pi}{32}}\right)=f\left(\lambda^{*}\right)=b-\left(r \lambda^{*}+k \lambda^{* 2}\right)$

where $k$ is constant and $r$ and $b$ are variable coefficients.

$b=\left(\frac{f_{T}}{3} \sin \theta+\frac{\pi f_{p}}{12} \sin 2 \theta\right) / I^{*}+\frac{\pi}{32}$

$r=D^{*}-\left(f_{R} \operatorname{sgn}(\lambda)-f_{T} \sin \theta\right) / 3$

$k=f_{R}\left(I^{*}+\frac{\pi}{32}\right) / 3$

According to Strogatz, (2014), in equation 5.1, the parenthesised phrase expresses prototypical of transcritical bifurcation while if $r=0$, the rest phrase forms a saddle node bifurcation. In transcritical bifurcation, the fixed point interchanges its stability with another fixed point as the parameter is varied (Strogatz, 2014). In other words, it is possible to find a threshold for a system in transcritical bifurcation. Fixed points of the parenthesised phrase in equation 5.1, i.e. $\left(r \lambda^{*}+k \lambda^{* 2}\right)$, are $\lambda=0$ and $\lambda=-r\left(I^{*}+\frac{\pi}{32}\right) / k$. Intersection of these lines in the stability diagram denotes threshold of the system, that is, the threshold is found if $r\left(I^{*}+\frac{\pi}{32}\right)=0$ which yields:

$\gamma_{T h}=\frac{\left(f_{R}-f_{T}\right)}{3}\left(I^{*}+\frac{\pi}{32}\right)$

where the values of $f_{R}$ and $f_{T}$ are found by the value of autorotation in equation 3.15, and $\gamma=D^{*}\left(\mathrm{I}^{*}+\frac{\pi}{32}\right)$. As demonstrated in figure 5-1, for $\gamma<\gamma_{T h}, \lambda=0$ denotes the unstable points and $\lambda=-r\left(I^{*}+\frac{\pi}{32}\right) / k$ corresponds to the stable points whereas, for $\gamma>\gamma_{T h}, \lambda=0$ represents the stable points and $\lambda=-r\left(I^{*}+\frac{\pi}{32}\right) / k$ gives the unstable points. Note that the points correspond to $\lambda=0$, when $\gamma \gg \gamma_{T h}$, is interpreted physically as a quasi-stationary plate, that is, the plate shows very small oscillation in the fluid flow because of the large value of $\mathrm{D}^{*}$. 


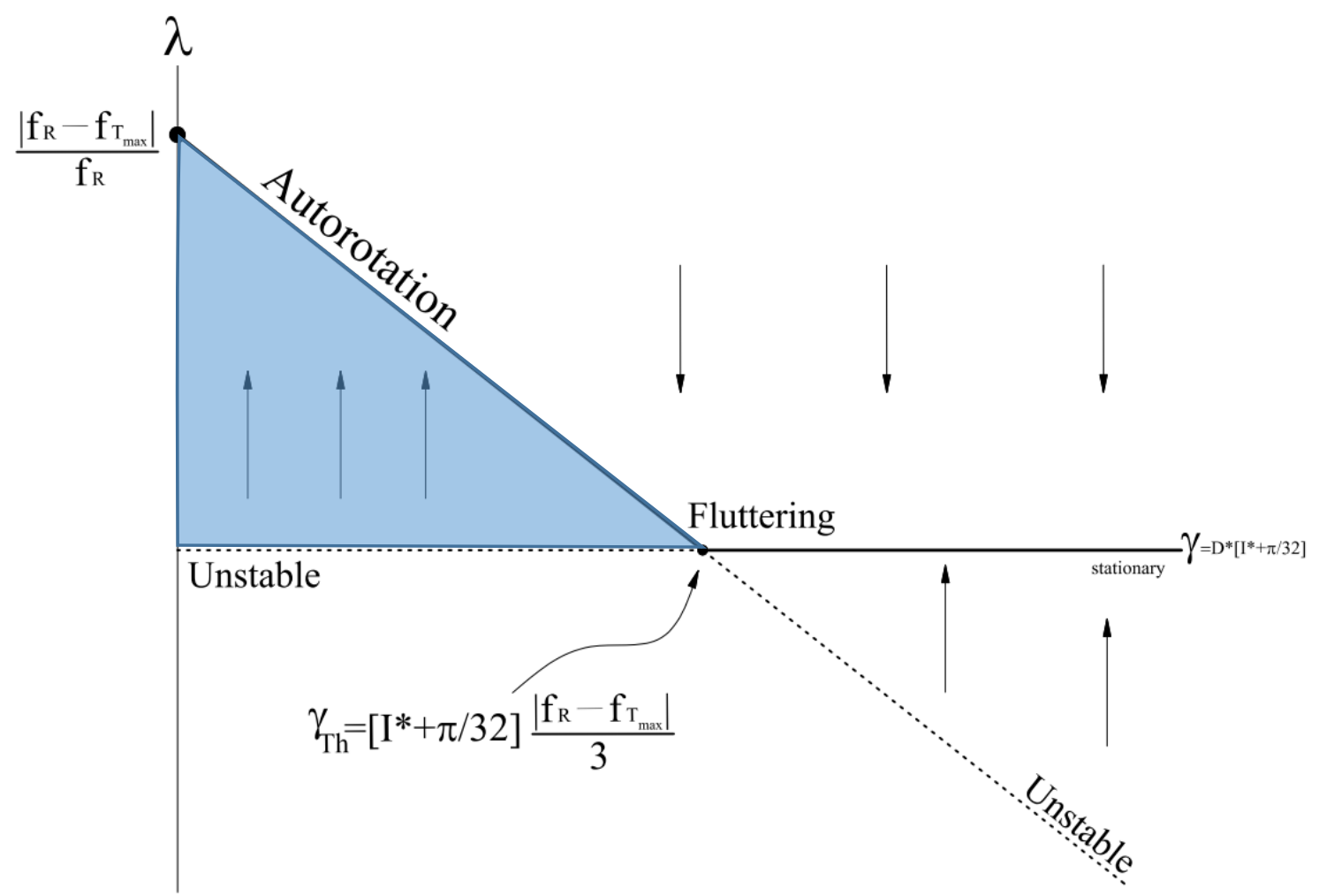

Figure 5-1: Stability diagram of transcritical bifurcation for equation 5.1. In this figure, $\gamma=D^{*}\left(I^{*}+\frac{\pi}{32}\right)$ and $\gamma_{\mathrm{Th}}$ is threshold of system. The dimensionless moment of inertia $\left(\mathrm{I}^{*}\right)$ and dimensionless damping $\left(\mathrm{D}^{*}\right)$ have been defined in equation 3.13 and coefficients of $f_{R}$ and $f_{T}$ are given by equation 3.15 .

The horizontal axis of figure 5-1 is labeled as $\gamma=D^{*}\left(I^{*}+\frac{\pi}{32}\right)$ where $\mathrm{D}^{*}$, see equation 3.13 , is the inverse proportion of the current velocity. The $\gamma$ shows that fluttering and autorotation are dependent on the moment of inertia and the current velocity. There is a critical value of $\mathrm{D}^{*}\left(\mathrm{D}^{*}\right.$ critical $)$ in which, for $D^{*}>D_{\text {critical }}^{*}$, the mode of rotation is fluttering and $D^{*} \gg D_{\text {critical }}^{*}$, the oscillation vanishes so that it supposes that the plate is stationary. $\mathrm{D}^{*}$ critical is independent of $\mathrm{I}^{*}$ and found as $D_{\text {critical }}^{*}=\left|f_{R}-f_{T_{\text {max }}}\right| / 3$. In absence of structural damping $\left(D^{*}=0\right)$, the plate reaches to an asymptotic tip speed ratio of $\lambda=$ $\left|f_{R}-f_{T_{\text {max }}}\right| / f_{R}$ which is independent of the moment of inertia or I*.

In figure 5-1, for $\gamma<\gamma_{T h}$, the points on stable line $\lambda=-r\left(I^{*}+\frac{\pi}{32}\right) / k$ represent autorotation phenomenon. It means that all points, in the around, are flown towards autorotation. In other words, the region between the horizontal unstable line and the inclined stable line (blued area in figure 5-1) denotes the possibility of a situation in which autorotation occurs. This region depends on $\mathrm{I}^{*}$ so that the decreasing $\mathrm{I}^{*}$ makes it diminishes and consequently reduces the possibility of happening autorotation. The blued area is enlarged when $\mathrm{I}^{*}$ increases, that is, autorotation occurs with a higher probability if $\mathrm{I}^{*}$ increases. The threshold $\left(\gamma_{T h}\right)$ is also dependent on $\mathrm{I}^{*}$ so that it tends toward zero by decreasing $\mathrm{I}^{*}$. Autorotation occurs in a broad range of $\mathrm{D}^{*}$ when $\gamma_{T h}$ increases. In other words, the chance of occurring autorotation for the high values of $I^{*}$ increases even at very low current velocity. Figure 5-2 illustrates the former discussion. 


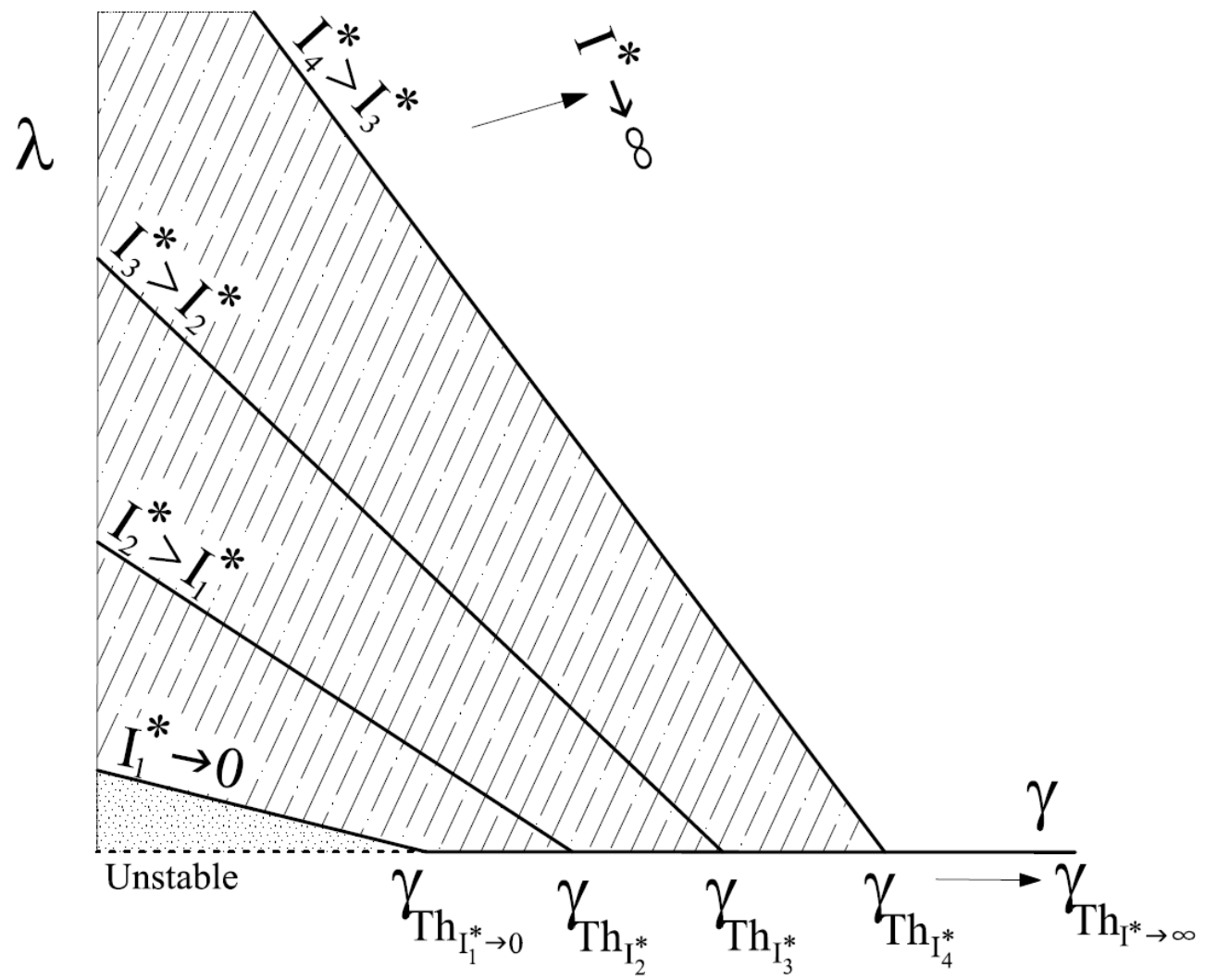

Figure 5-2: Threshold of the system as a function of $I^{*}$. The hatched area for each $\mathrm{I}^{*}$ denotes the possibility of occurring autorotation.

On the other side, if let $r=0$ in equation 5.1, the rest of equation makes a normal form of the saddle node bifurcation which the fixed points are $\lambda= \pm\left(I^{*}+\frac{\pi}{32}\right) \sqrt{b / k}$ and exist when $\mathrm{b} \geq 0$. Given the values of $f_{\mathrm{p}}, f_{\mathrm{R}}$ and $f_{\mathrm{T}}$ by equation 3.13 and the definition of $\mathrm{b}$ by equation 3.15 , the roots of $b=0$ in the range of zero and $\pi(0 \leq \theta \leq \pi)$ are $\theta=0,1.66, \pi$ in radian. Among these points, $\theta=1.66 \mathrm{rad}$ represents the unstable point and two others are the stable points. The unstable point refers an angle at which the plate tends to oscillate around. In other words, fluttering in such a system is done around the angle of $\pi / 2$, that is, fluttering occurs in situation almost perpendicular to the current. Figure 5-3 provides a comparison between the result of equation 3.12 and experimental data for the unstable point of saddle node bifurcation. As can be seen, in both figures, the plate fluttering reaches the angle approximately $1.66 \mathrm{rad}$ and therefore the calculated points are verified.
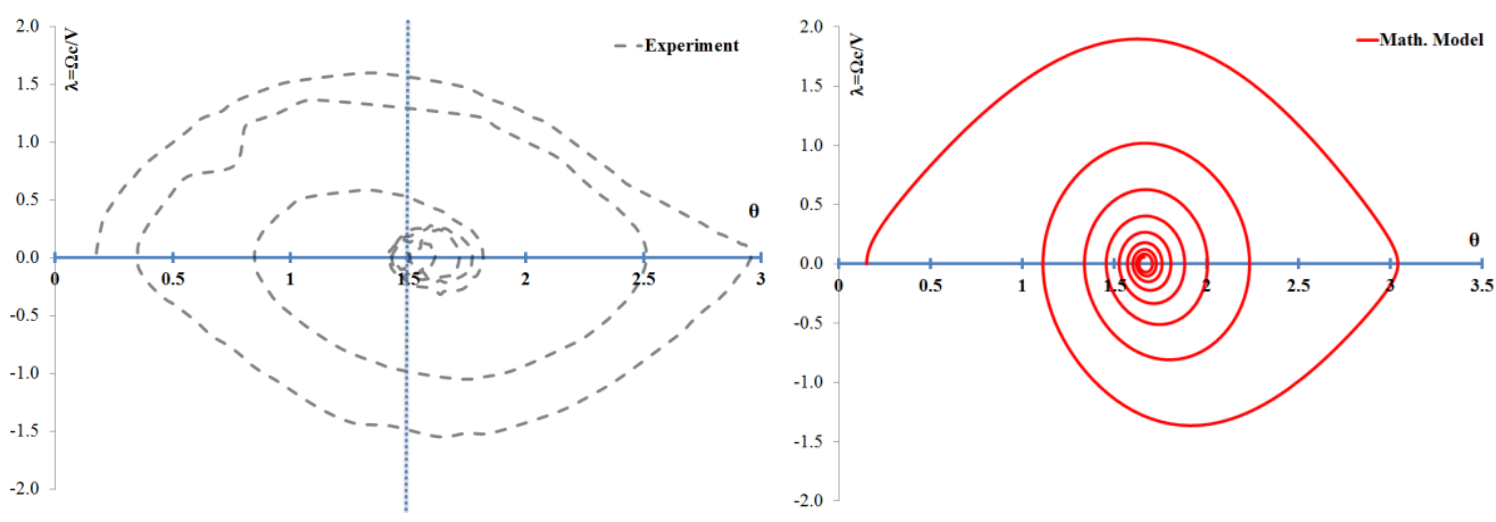
Figure 5-3: Comparison of phase diagram of experimental data and mathematical model for $I^{*}=0.0323$ and $\mathrm{D}^{*}=0.0134$. The stable fixed point in both diagrams is approximately same and a bit more than $\pi / 2$. In the left diagram (experiment), vertical dotted line indicates location of $\pi / 2$.

In more general comparison, we have tried to present a bifurcation diagram in which the type of dynamics in different $I^{*} \mathrm{~s}$ and current velocities has been determined analytically and compared with experimental results. To do this, assuming the autorotation occurs when the plate rotates at least one cycle. Accordingly, it is possible to formulize this expression as $\int_{\theta_{1}}^{\theta_{1}+2 \pi} \ddot{\theta}(\theta, \dot{\theta}(\theta)) d \theta=0$ (Rostami and Fernandes, 2018). Suppose that $\theta_{1}=0$ and $\dot{\theta}=$ $\Theta|\sin \theta|$, using equation 3.12 and applying aforementioned definition for autorotation, the following bifurcation diagram (figure 5-4) can be achieved for different $I^{*}$ s and velocities. Figure 5-4 shows a very good level of accuracy and applicability of this analysis.
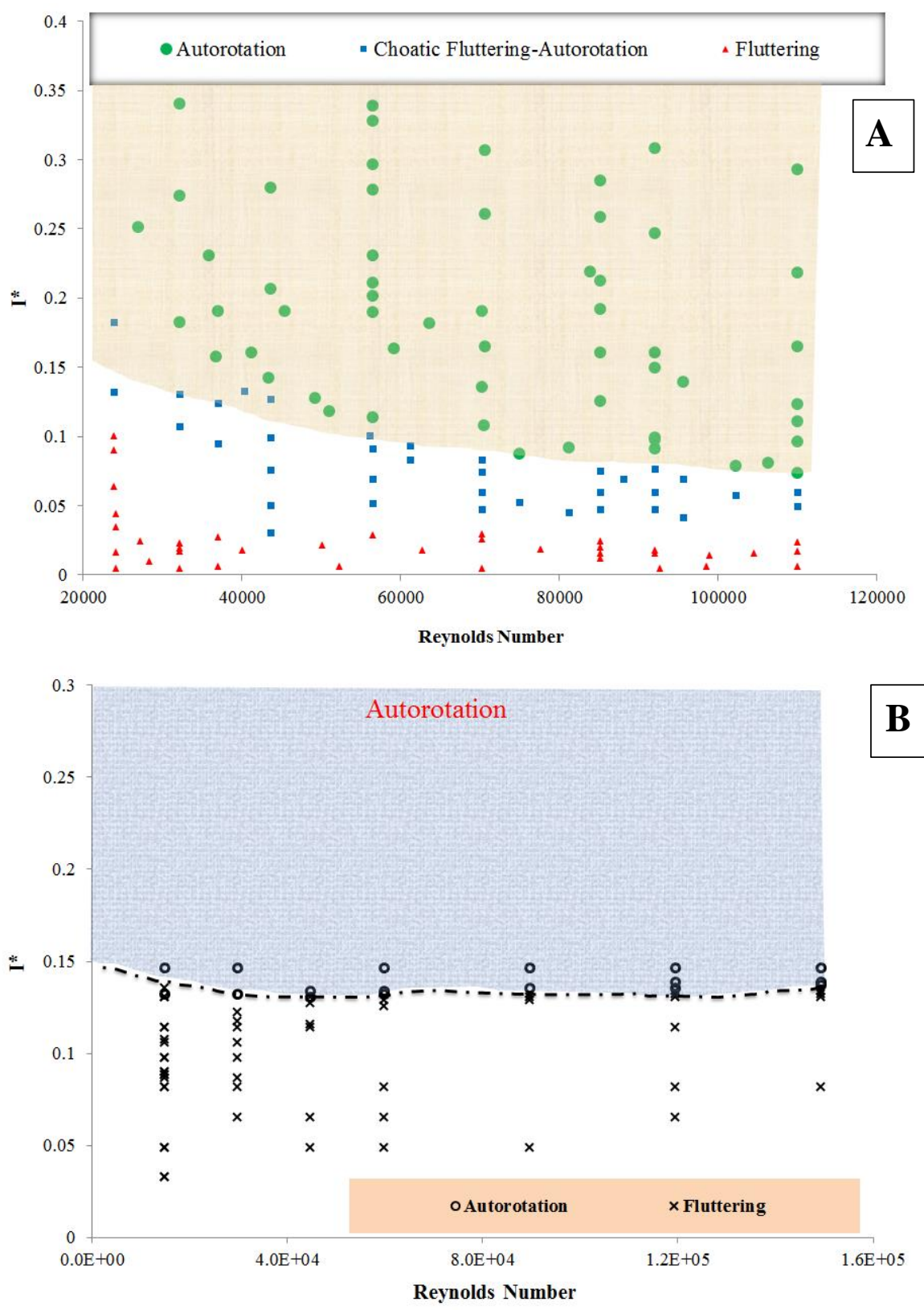

Figure 5-4: Bifurcation diagrams of I* versus Reynolds number A: Experimental data (Bakhshandeh rostami, 2016), B: results of the analytical model. 


\section{Conclusion remark}

This paper aims to understand the various portions of torque on a rotating non-circular body through the fluid current. A theoretical approach was employed in this paper which is named far-field method. In this method, forces and moment applied on the body are derived from the integral balance of the momentum equations. Using Gauss and Stokes theorems, the spatial integrals (volume integral) change to surface integral which provides many facilities to analyzing the external flow problems. The total torque over a non-circular body through the current was obtained by far-field method which is composed of torques due to the helicity, the shear stress, the pressure distribution, the stress due to deformation and also the vorticity variation. For a simple physics such as a $2 \mathrm{D}$ rotating rigid body, this complex expression is simplified to two components as the moment due to the pressure distribution and the moment generated by the vorticity field. In the current paper, these two components were analytically defined for a $2 \mathrm{D}$ rotating rigid flat plate.

Two components of the circulation of a rotating body through the current are the portion corresponds to the angular velocity and the part relates to the current velocity. The last one obtains by the Kutta-Joukowski condition. The rotational part of circulation relies upon the direction of rotation. Accordingly, the circulation of the rotation for fluttering includes the negative and the positive values and it becomes small by the time because of the dissipating of the oscillation.

The torque on a 2D rotating plate comes from three effects. These effect are included the current effect, the rotational effect and the Magnus effect. The last one is the contribution of the current velocity and the plate angular velocity, simultaneously. Magnus torque includes both effects as the excitation of the rotation and the prevention of the rotation, depends on the value. The rotational part is ever negative and therefore is a resisting torque for the rotation. The rotation will continue if an additional and sufficient large torque, which is provided by moment of inertia, can overcome the resisting torque. Thus, autorotation occurs when sufficient moment of inertia is provided for the system.

Stability analysis divulged that such a system has transcritical bifurcation overall, while saddle node bifurcation will happen in specific conditions. In special value of dimensionless structural damping, which is called $\mathrm{D}^{*}$ critical, the saddle node bifurcation will happen. In this condition, depends on $I^{*}$, either autorotation or fluttering occurs. For transcritical bifurcation, a threshold $\left(\gamma_{T h}\right)$ has been defined which is dependent on $\mathrm{I}^{*}$. Autorotation occurs in a broad range of $\mathrm{D}^{*}$ if $\gamma_{T h}$ increases. In other words, the chance of occurring autorotation for the high values of $\mathrm{I}^{*}$ increases even at very low current velocity.

The authors acknowledge greatly CNPq (The Brazilian National Research Council), and LOC/COPPE/UFRJ (Laboratory of Waves and Current of COPPE, Federal University of Rio de Janeiro).

\section{References}

ROUSSEAUX, G., SEIFER, S., STEINBERG, V., \& WIEBEL, A. 2007 On the Lamb vector and the hydrodynamic charge. Exp. Fluids, 42(2), 291-299. 
MELE, B., \& TOGNACCINI, R. 2014 Aerodynamic force by Lamb vector integrals in compressible flow. Phys. Fluids, 26 (5), 056104.

ARMANDEI, M., FERNANDES, A. C., \& ROSTAMI, A. B. 2016 Hydroelastic Buffeting Assessment Over a Vertically Hinged Flat Plate. Experimental Techniques, 40(2), 833-839.

WU, J. Z., LU, X. Y., \& ZHUANG, L. X. 2007 Integral force acting on a body due to local flow structures. J. Fluid Mech., 576, 265-286.

ROSTAMI, A. B., GHADIMI, P., \& GHASEMI, H. 2016 Adaptive viscous-inviscid interaction method for analysis of airfoils in ground effect. Journal of the Brazilian Society of Mechanical Sciences and Engineering, 38(6), 1593-1607.

SAMADPOUR, M., ASADI, H., \& WANG, Q. 2016 Nonlinear aero-thermal flutter postponement of supersonic laminated composite beams with shape memory alloys. European Journal of Mechanics-A/Solids, 57, 18-28.

MEHRI, M., ASADI, H., \& WANG, Q. 2016 On dynamic instability of a pressurized functionally graded carbon nanotube reinforced truncated conical shell subjected to yawed supersonic airflow. Composite Structures, 153, 938-951.

ASADI, H., \& WANG, Q. 2017a An investigation on the aeroelastic flutter characteristics of FG-CNTRC beams in the supersonic flow. Composites Part B: Engineering, 116, 486499.

GHADIMI, P., ROSTAMI, A. B., \& JAFARKAZEMI, F. 2012 Aerodynamic analysis of the boundary layer region of symmetric airfoils at ground proximity. Aerospace Science and Technology, 17(1), 7-20.

SAFFMAN, P.G. 1992 Vortex Dynamics. Cambridge University Press, Cambridge.

WU, J.C., 2005 Elements of Vorticity Aerodynamics. Tsinghua University Press, Beijing.

WU, J.-Z., MA, H.-Y., \& ZHOU, M.-D., 2006 Vorticity and Vortex Dynamics. Springer, New York.

YOSHIDA, Z., MORRISON, P.-J., 2016 Epi-two-dimensional flow and generalized enstrophy. arXiv:1604.02339.

MOFFATT, H. K. 2014 Helicity and singular structures in fluid dynamics. Proc. National Acad. of Sciences, 111(10), 3663-3670.

XIA, X., \& MOHSENI, K. 2013 Lift evaluation of a two-dimensional pitching flat plate. Phys. Fluids, 25(9), 091901.

MARONGIU, C., \& TOGNACCINI, R. 2010 Far-field analysis of the aerodynamic force by lamb vector integrals. J. AIAA, 48(11), 2543-2555.

YANG, Y. T., ZHANG, R. K., AN, Y. R., \& WU, J. Z. 2007 Steady vortex force theory and slender-wing flow diagnosis. Acta Mech. Sinica, 23(6), 609-619.

PESAVENTO, U., \& WANG, Z. J. 2004 Falling paper: Navier-Stokes solutions, model of fluid forces, and center of mass elevation. Phys. Rev. Lett. 93 (14), 144501. 
THOMSON, J. J. 1883 A Treatise on the Motion of Vortex Rings. Macmillan, London.

LAMB H., 1945 Hydrodynamics. $6^{\text {th }}$ edition, Dover publication.

BATCHELOR, G. K. 1967 An Introduction to Fluid Dynamics. Cambridge University Press.

LiGHTHILL, M. J. 1986 An Informal Introduction to Theoretical Fluid Dynamics. Oxford University Press.

WU, J. Z., \& WU, J. M. 1996 Vorticity dynamics on boundaries. Adv. App. Mech. 32, 119275.

ROSTAMI, A. B., \& FERNANDES, A. C. 2015a The effect of inertia and flap on autorotation applied for hydrokinetic energy harvesting. Appl. Energy 143, 312-323.

FERNANDES, A. C., \& ROSTAMI, A. B. 2015 Hydrokinetic energy harvesting by an innovative vertical axis current turbine. Rene. Energy 81, 694-706.

FERNANDES, A. C., ROSTAMI, A. B., CANZIAN, L. G., \& SEFAT, S. M. 2013 Vertical axis current turbine (VACT) and its efficiency. In ASME 2013 32nd International Conference on Ocean, Offshore and Arctic Engineering(pp. V008T09A051V008T09A051). American Society of Mechanical Engineers.

ROSTAMI, A. B., \& FERNANDES, A. C. 2014 Plate shape effect on the performance of the vertical axis auto rotation current turbine (VAACT). In ASME 2014 33rd International Conference on Ocean, Offshore and Arctic Engineering (pp. V09AT09A041-V09AT09A041). American Society of Mechanical Engineers.

ROSTAMI, A. B., \& FERNANDES, A. C. 2015b Simulation of Fluttering and Autorotation Motion of Vertically Hinged Flat Plate. In ASME 2015 34th International Conference on Ocean, Offshore and Arctic Engineering (pp. V001T01A001-V001T01A001). American Society of Mechanical Engineers.

ROSTAMI, A. B., \& ARMANDEI, M. 2017 Renewable energy harvesting by vortex-induced motions: review and benchmarking of technologies. Renewable and Sustainable Energy Reviews, 70, 193-214.

MARTINEZ-VAZQUEZ, P., BAKER, C. J., STERLING, M., QUINN, A., \& RICHARDS, P. J. 2010 Aerodynamic forces on fixed and rotating plates. Wind Struct. 13 (2), 127-144.

SMITH, E. H. 1971 Autorotating wings: an experimental investigation. J. Fluid Mech. 50, 513-534.

STROGATZ, S. H. 2014 Nonlinear dynamics and chaos: with applications to physics, biology, chemistry, and engineering. Westview press.

WANG, S., ZHANG, X., HE, G., \& LIU, T. 2013 A lift formula applied to low-Reynoldsnumber unsteady flows. Phys. Fluids, 25(9), 093605.

MILNE-THOMPSON, L. M. 1968 Theoretical hydrodynamics. London: MacMillan, 1968, 5th ed. 
SAlEHI, M., GHADIMI, P., \& ROSTAMI, A. B. 2014 A more robust multiparameter conformal mapping method for geometry generation of any arbitrary ship section. J. Eng. Math. 89(1), 113-136.

MAHADEVAN, L. 1996. Tumbling of a falling card. Contes Rendues (Academy of Sciences, Paris) Series 2b 323:729.

TANABE, Y., \& KANEKO, K. 1994 Behavior of a falling paper. Phys. Rev. Lett., 73(10), 1372.

BELMONTE, A., EISENBERG, H., \& MOSES, E. 1998 From flutter to tumble: inertial drag and Froude similarity in falling paper. Phys. Rev. Lett. 81(2), 345.

SEDOV, L. I. 1980 Two-dimensional problems of hydrodynamics and aerodynamics. Moscow Izdatel Nauka.

LOMAX H., PUlliam T.H., ZINGG D.W. 2001 Fundamental of Computational Fluid Dynamics, Springer Berlin Heidelberg.

FIELD S. B., KLAUS M., MOORE M. G., NORI F. 1997 Chaotic dynamics of falling disks. Nature, 388, 252-254.

IVERSEN, J. D. 1979 Autorotating flat-plate wings: the effect of the moment of inertia, geometry and Reynolds number. J. Fluid Mech. 92(02), 327-348.

LENTINK, D., DICKSON, W. B., Van Leeuwen, J. L., \& Dickinson, M. H. (2009). Leadingedge vortices elevate lift of autorotating plant seeds. Science, 324(5933), 1438-1440.

KUZNETSOV, S. P. 2015 Plate falling in a fluid: Regular and chaotic dynamics of finitedimensional models. Regular and Chaotic Dynamics, 20(3), 345-382.

ANDERSEN, A., PESAVENTO, U., \& WANG, Z. 2005 Analysis of transitions between fluttering, tumbling and steady descent of falling cards. Journal of Fluid Mechanics, 541, 91-104.

WILLMARTH, W. W., HAWK, N. E., \& HARVEY, R. L. 1964 Steady and Unsteady Motions and Wakes of Freely Falling Disks. Physics of Fluids, 7, 197-208.

SKEWS, B. W. 1990 Autorotation of rectangular plates. Journal of Fluid Mechanics, 217, 3340.

Wu, J. C. 1981 Theory for aerodynamic force and moment in viscous flows. AIAA J. 19, 432441.

Wu, J. C. 2005 Elements of Vorticity Aerodynamics. Tsinghua University Press, Beijing.

COPELAND, G. S. (1994). A perturbed-pendulum model for flat-plate autorotation. Journal of fluids and structures, 8(2), 125-138.

TRChAlíK, J., GILliES, E. A., \& THOMSON, D. G. 2008 Development of an Aeroelastic Stability Boundary for a Rotor in Autorotation.

LUGT, H. J. 1983 Autorotation. Annual Review of Fluid Mechanics, 15(1), 123-147. 
BAKHSHANDEH ROSTAMI, A. 2015 Energy Harvesting By Hydro/Aero Elastic Phenomena in Small Scale. 83-110.

ASADI, H., \& WANG, Q. 2017 b Dynamic stability analysis of a pressurized FG-CNTRC cylindrical shell interacting with supersonic airflow. Composites Part B: Engineering, 118, 15-25.

ASADI, H., SOURI, M., \& WANG, Q. 2017 A numerical study on flow-induced instabilities of supersonic FG-CNT reinforced composite flat panels in thermal environments. Composite Structures, 171, 113-125.

ROSTAMI, A. B., \& FERNANDES, A. C. 2018 Mathematical model and stability analysis of fluttering and autorotation of an articulated plate into a flow. Communications in Nonlinear Science and Numerical Simulation, 56, 544-560.

ROSTAMI, A. B., \& FERNANDES, A. C. 2017 From fluttering to autorotation bifurcation of a flat plate in a current. Journal of the Brazilian Society of Mechanical Sciences and Engineering, 39(11), 4769-4784.

Bakhshandeh rostami A. Hydrokinetic energy harvesting by autorotation of a plate with hinged axis PhD Thesis. UFRJ; 2016. 